\title{
A High-content Screening Assay based on Automated Microscopy for Monitoring Antibiotic Susceptibility of Mycobacterium Tuberculosis Phenotypes
}

\section{Sadaf Kalsum}

Linköping University

Blanka Andersson

Linköping University

Jyotirmoy Das

Linköping University

Thomas Schön

Linköping University

Maria Lerm ( $\nabla$ maria.lerm@liu.se )

Linköping University

\section{Research Article}

Keywords: cording, planktonic, Mycobacterium tuberculosis, whole cell screening, automated live-cell imaging

Posted Date: January 7th, 2021

DOI: https://doi.org/10.21203/rs.3.rs-133359/v1

License: (c) (i) This work is licensed under a Creative Commons Attribution 4.0 International License.

Read Full License 


\section{A high-content screening assay based on automated microscopy for}

2 monitoring antibiotic susceptibility of Mycobacterium tuberculosis

3 phenotypes

4

5 Sadaf Kalsum ${ }^{1 *}$, Blanka Andersson ${ }^{1 *}$, Jyotirmoy Das ${ }^{1}$, Thomas Schön ${ }^{2}$ and Maria Lerm ${ }^{1}$

6

7

$8 \quad{ }^{1}$ Division of Inflammation and Infection, ${ }^{2}$ Division of Clinical Microbiology

9 Department of Biomedical and Clinical Sciences, Faculty of Medicine and Health Sciences, Linköping University, SE-58185, Linköping, Sweden.

*) Shared first authorship

Corresponding author:

Maria Lerm, Professor in Medical Microbiology

Div. of Inflammation and Infection, Lab 1, floor 12

Dept. of Biomedical and Clinical Sciences, Faculty of Medicine and Health Sciences

Linköping University

SE-58185 Linköping, Sweden

Phone: +46-732707786

E-mail: maria.lerm@liu.se 


\section{Abstract}

22

\section{Background}

Assays enabling efficient high throughput drug screening are necessary for the discovery of new anti-mycobacterial drugs. The purpose of our work was to develop and validate an assay based on live-cell imaging which can monitor growth of two distinct phenotypes of Mycobacterium tuberculosis and to test their susceptibility to commonly used TB drugs.

\section{Results}

Both planktonic and cording phenotypes were successfully monitored as fluorescent objects using the live-cell imaging system Incucyte S3, allowing collection of data describing distinct characteristics of aggregate size and growth. The quantification of changes in total area of aggregates was used to define IC50 and MIC values of selected TB drugs which revealed that the cording phenotype grew more rapidly and displayed a higher susceptibility to rifampicin. A checkerboard approach, testing pair-wise combinations of sub-inhibitory concentrations of drugs, revealed rifampicin, linezolid and pretomanid as superior in inhibiting growth of cording phenotype.

\section{Conclusion}

Our results emphasize the efficiency of using automated live-cell imaging and its potential in high-through put whole-cell screening to evaluate existing and search for novel antimycobacterial drugs.

\section{Keywords}

cording, planktonic, Mycobacterium tuberculosis, whole cell screening, automated live-cell imaging 


\section{Background}

Tuberculosis (TB) stands out as one of the most prevalent disease worldwide with a medication period largely extending that of other bacterial infections. The treatment of TB requires 6-9 months of chemotherapy with multiple drugs and is hampered by spread of antibiotic resistance, narrowing the therapeutic options. The conventional anti-TB drugs have been in use for many decades and the need to broaden their palette is urgent, pushed by an increase in incidence of multidrug-resistant and extensively drug-resistant TB cases. The search for new treatment regimens has resulted in the identification of several candidates, including new compounds or repurposed drugs (1).

Prolonged treatment and variable susceptibility to antibiotics can be attributed to the heterogeneity of populations of Mycobacterium tuberculosis (Mtb, the causative agent of TB), which exists in diverse phenotypes both in vitro and in vivo. The inherent ability of Mtb to form organized aggregates has been known for several decades (2) and has been often related to virulence. Cording mycobacteria grow aligned into tight bundles, where the orientation of the long axis of each bacterial cell within the cord is parallel to the long axis of the cord $(3,4)$. As we have previously demonstrated, the cording phenotype represents more intrusive interaction with immune cells causing macrophages to release macrophage extracellular traps (METs) (5). Recently, the cording phenotype has been shown to cause extensive immunopathological changes associated with active $\mathrm{TB}$ in $\mathrm{C} 3 \mathrm{HeB} / \mathrm{FeJ}$ mice (6). Mtb cords were also identified inside human alveolar macrophages obtained from patients with active TB (7) and lymphatic endothelial cells isolated from patients with extrapulmonary TB (8).

Phenotypic drug screening based on the response of growing bacterial cultures allows the identification of a broader spectrum of inhibitors regardless on mechanism of their action. This so-called "whole-cell screening" has the advantage over target-based screening that only 
molecules that can penetrate and actually kill/prevent growth of bacteria are selected as hits ( 9 , 10). This approach led to the discovery of bedaquilin, one of the few recently introduced drugs (11). More efficient methods based on reporter strains, such as H37Rv carrying bioluminescent or fluorescent genes, has facilitated the discovery of several lead compounds (12). Due to the phenotypic heterogeneity of mycobacteria, the need for combination of several anti-microbial drugs with different modes of action will continue to be a cornerstone in TB treatment. Even if all possible combinations cannot be tested, high throughput assays allowing pairwise combinations can generate essential information for mathematical models to predict high-order interactions (13). Image-based assays bring another dimension into drug screening and if automated imaging systems are used with live cultures, data reflecting the growth kinetics and morphological appearance of the models can be feasibly collected. Their power for drug discovery based on investigating the intracellular fate of Mtb treated with anti-microbial compounds has been demonstrated in multiple studies (14-18) but to our knowledge no imaging assays analysed the growth of axenic mycobacterial cultures.

Since there is evidence that cording represents an important intracellular phenotype and considering the potential importance of various phenotypes in compound discovery, we developed and validated an assay based on automated live-cell imaging to monitor growth of two distinct phenotypes of Mtb and used it in a checkerboard approach to analyse the effect of combinations of commonly used as well as recently developed TB drugs.

\section{Results}

Distribution of aggregates sizes reflects the bacterial phenotype and is altered during bacterial growth 
In line with our previous findings (5), planktonic bacteria (originating from standing cultures in Middlebrook 7H9 broth with Tween-80) grew as small, dispersed aggregates (Fig. 2A, day2), while the cording phenotype (obtained from shaken cultures in Middlebrook $7 \mathrm{H} 9$ broth without Tween-80) formed more organized structures characteristic for mycobacterial cording (Fig. 2B, day-2). The phenotypic differences persisted throughout the experiment although the growth conditions were identical for both phenotypes (Tween-80-free cell culture medium) from the initiation of the experiment (Fig. 2A-B). In a parallel experiment, bacteria originating from standing cultures were seeded in wells with Middlebrook $7 \mathrm{H} 9$ broth with or without Tween-80. Again, the absence of Tween-80 promoted the cording phenotype (Fig. 2C). We reasoned that use of cell culture medium, in our case Dulbeccos's Modified Eagle Medium (DMEM) with human serum instead of conventional broth could be an advantage if our assay would be later optimized for drug screening using infected human cells. The fluorescent bacterial aggregates were than classified by size and their number in each category was summarized in frequency plots. Day 0 in experiments was defined as a time point when antibiotics were added to the bacterial suspension. In the freshly harvested cultures (day -2), the planktonic bacteria contained a significantly higher number of small aggregates between 5$100 \mu \mathrm{m}^{2}$ as compared to the cording phenotype (Fig. 2D, Additional file 1: Table S1). On day 5 both phenotypes showed an increase in frequency in larger categories due to the growth of bacteria as enlarging aggregates rather than as separated cells. The planktonic phenotype contained significantly more smaller aggregates (between 10 and $500 \mu \mathrm{m}^{2}$ ) and similarly to day -2 , there were no aggregates larger than $1000 \mu \mathrm{m}^{2}$, whereas the cording phenotype contained aggregates in all larger categories (Fig. 2E, Additional file 1: Table S1). 

models. H37Rv growing in DMEM as planktonic (A) and cording (B) bacteria are shown at different time points. H37Rv grown in broth with or without Tween-80 (Tween) as indicated (C). Frequency plots of the distribution of aggregate sizes at day -2 (D) and day 5 (E). Columns represent size intervals and are logarithmically distributed up to $10^{4} \mu \mathrm{m} 2$. NP (non-present) marks intervals where no objects were identified. Data are presented as mean $\pm \mathrm{SD}(\mathrm{N}=3)$.

A

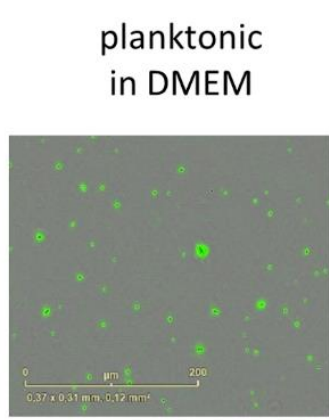

day-2

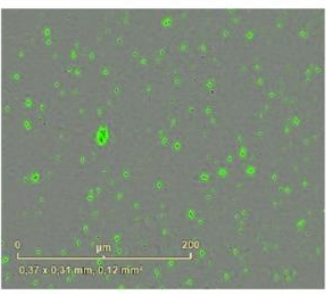

day0

day3
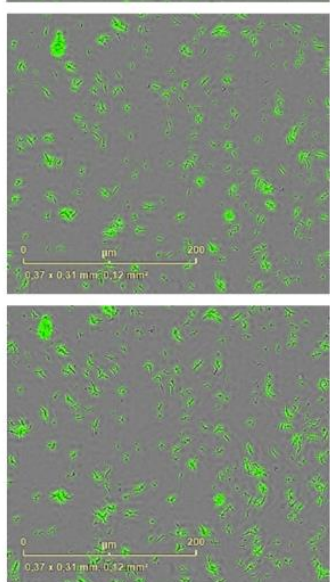

day5
B cording

in DMEM
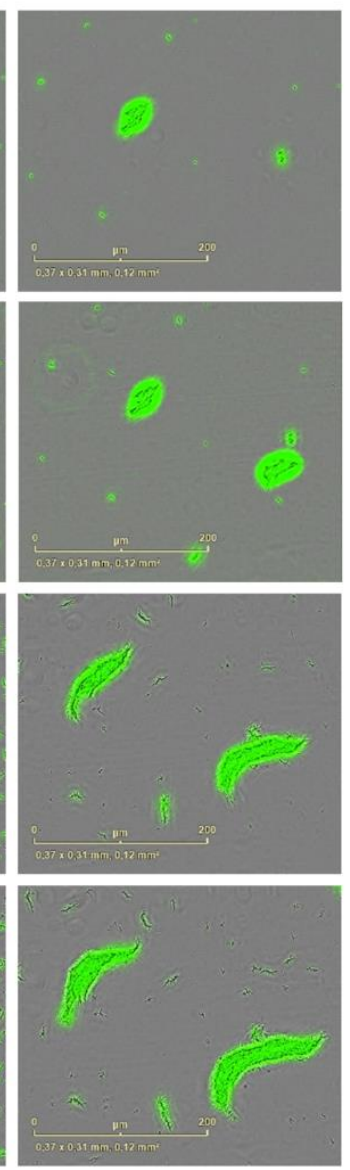

C

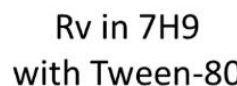

$\mathrm{Rv}$ in $7 \mathrm{H} 9$ without Tween-80
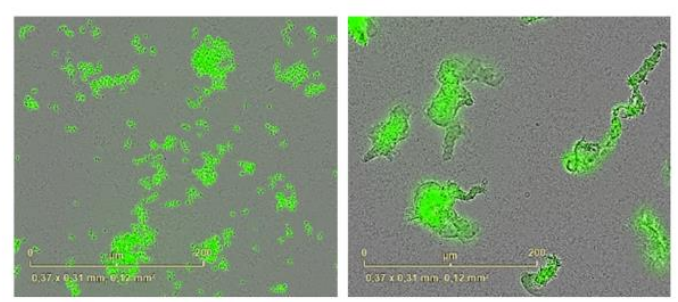

D
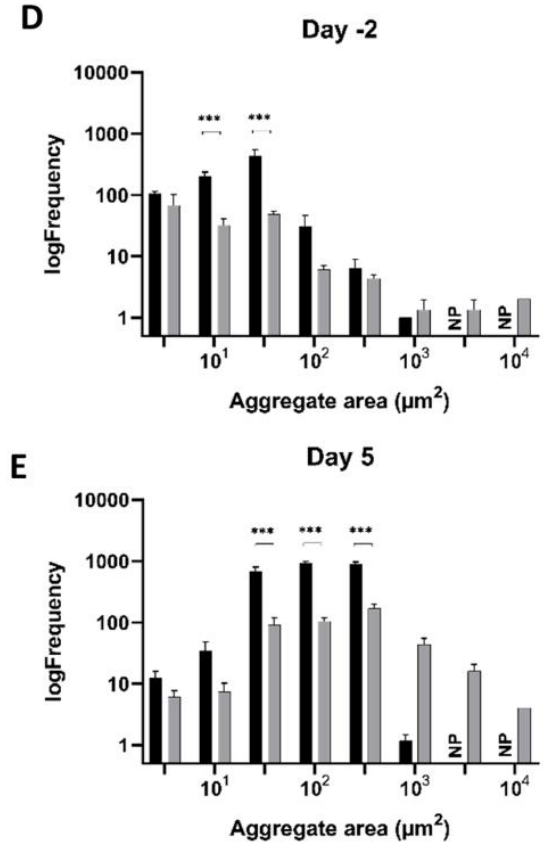

- planktonic

cording

We also investigated possibility to visualize the continuous changes in area of single aggregates. 
125 were analyzed through the MATLAB image processing tool to follow up the growth of

126 individual aggregates (Additional file 8: Movie S1).

\section{Distribution of aggregate sizes reflects antibiotic exposure}

128 In order to investigate whether the distribution of aggregates sizes could be affected by 129 antibiotic treatment, we treated the cultures with different concentrations of rifampicin (RIF).

130 At the lowest concentration $\left(1 \times 10^{-6} \mu \mathrm{g} / \mathrm{ml}\right)$, the frequency of larger aggregates increased 131 significantly with time whereas frequency of smaller aggregates significantly decreased (Fig. $1323 \mathrm{E}, \mathrm{F}$ ) comparably to the growth of untreated H37Rv (Fig. 3G, H). In contrast, the frequency 133 of aggregates in wells treated by highest concentration of RIF $(10 \mu \mathrm{g} / \mathrm{ml})$ remained unchanged 134 with time (Fig. 3A, B). A moderate shift in frequencies was observed in wells treated with an 135 intermediate dose of RIF $\left(1 \times 10^{-3} \mu \mathrm{g} / \mathrm{ml}\right.$, Fig. 3C, D). Similar results were observed for bacteria 136 treated with isoniazid (INH) (Fig. 4 A-H). 
147 (RIF). A, C, E) Frequency plots summarizing data from planktonic phenotype and B, D, F) 148 data from cording phenotype exposed to different concentrations of rifampicin after 3 and 5 149 days of incubation. G, H) Frequency plots of untreated controls for planktonic and cording 150 phenotype respectively. Data are presented as mean $\pm \mathrm{SD}(\mathrm{N}=3)$.
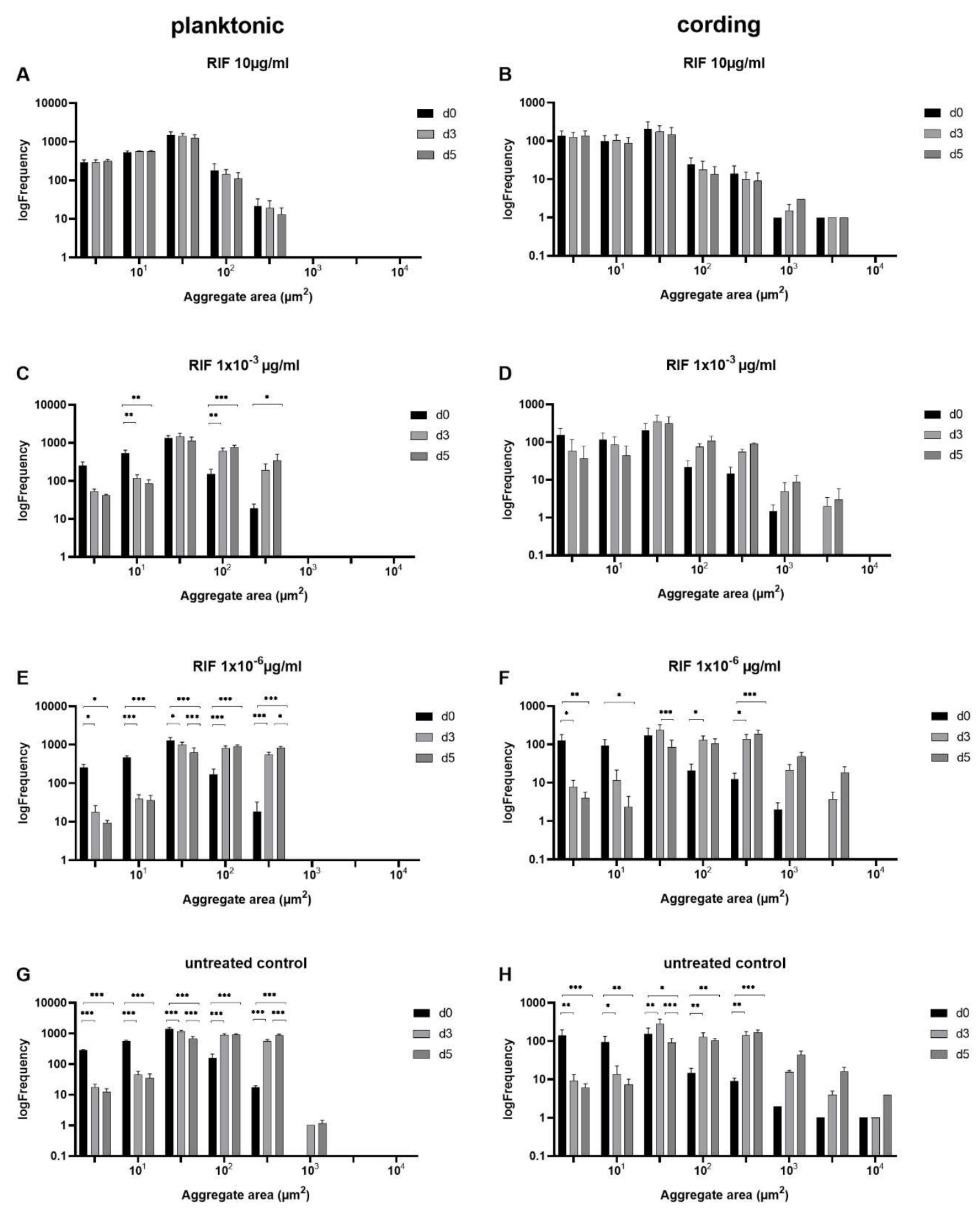

respectively. Data are presented as mean $\pm \mathrm{SD}(\mathrm{N}=3)$.

planktonic

A

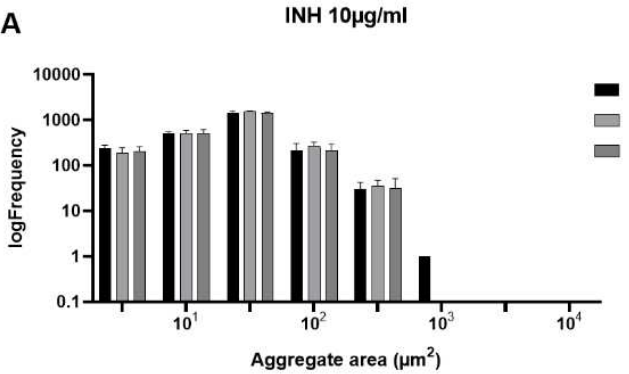

C

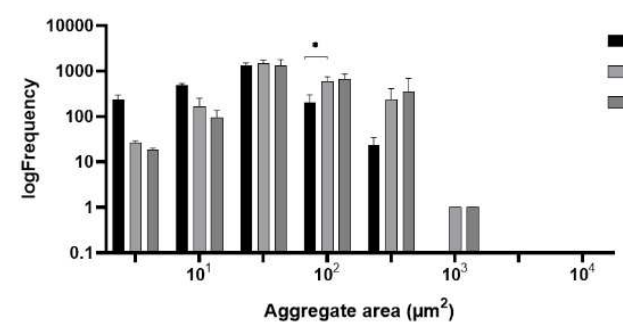

E

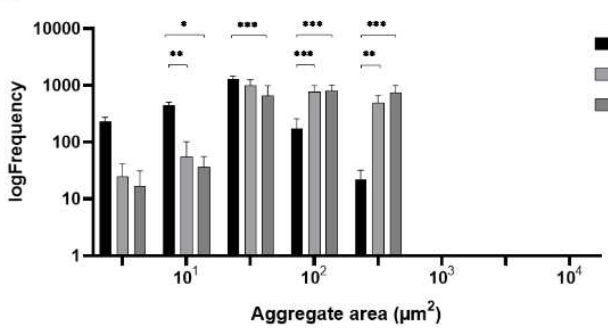

G

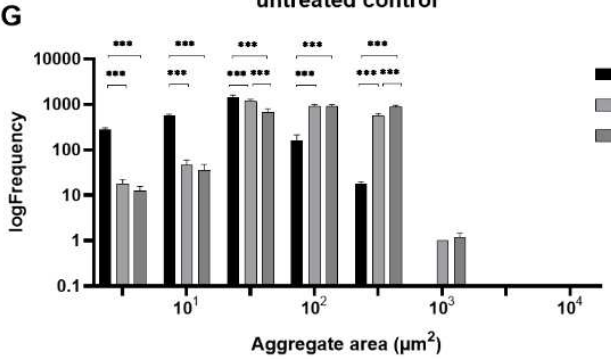

B - $\mathrm{d} 0$
$\mathrm{~d} 3$
$\mathrm{~d} 5$ $\square \mathrm{d} 3$

D

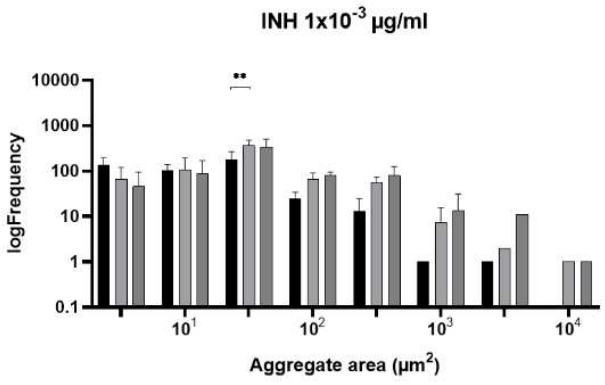

F $\mathrm{d} 0$
$\mathrm{~d} 3$
$\mathrm{~d} 5$ $\mathrm{d} 0$
$\mathrm{~d} 3$
$\mathrm{~d} 5$

H

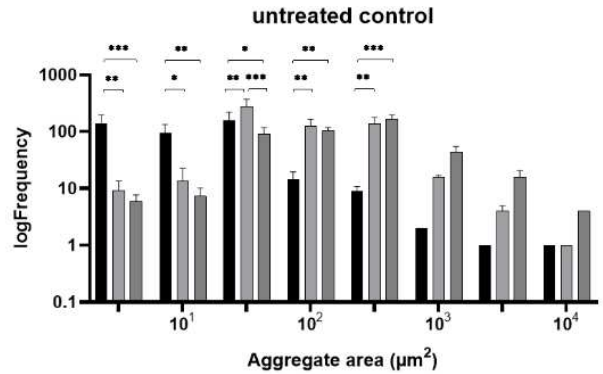

- do $\square \mathrm{d} 3$

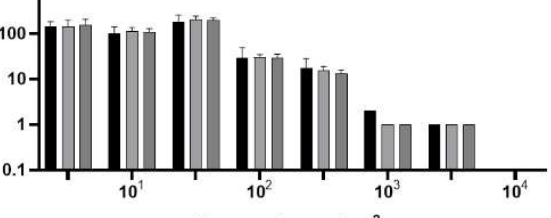

Aggregate area $\left(\mu \mathrm{m}^{2}\right)$

$\mathrm{d} 0$
$\mathrm{~d} 3$ $\square \mathrm{d} 5$

do
$\mathrm{d} 3$

d5

$\mathrm{d} 0$
$\mathrm{~d} 3$

$\square \mathrm{d} 5$

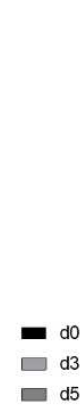

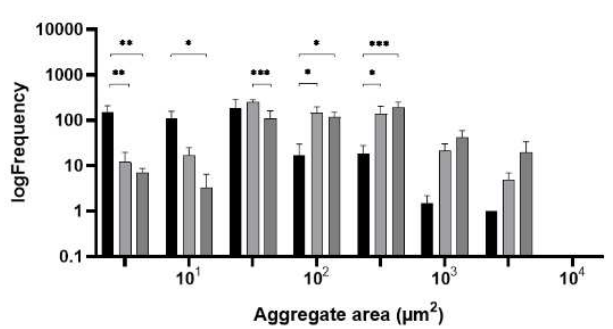

ggregate area $\left(\mu \mathrm{m}^{2}\right)$

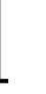



antibiotics

Next, to validate the usefulness and accuracy of the method, we set out to determine the Inhibitory Concentration 50 (IC50) and Minimal Inhibitory Concentration (MIC) of RIF and INH and other first- and second-line TB antibiotics (Table 1) for the respective phenotype. As the initial inoculum and growth rate differed between the two phenotypes (Additional file 2: Fig S1), we normalized the growth after antibiotic treatment to the untreated controls for each phenotype separately (Additional files 3-6: Fig. S2-5) after establishing that growth measurements of untreated bacteria performed with reliable reproducibility. Intra-assay variability at day 5 was $6,1 \%, 16,1 \%$ and $8,2 \%$ for the planktonic and $10,6 \%, 13,6 \%$ and $17,8 \%$ for the cording phenotypes, respectively, in each of three experiments. Inter-assay variability based on data from all three experiments was $10,1 \%$ for the planktonic and $19,9 \%$ for the cording model (Additional file 7: Table S2).

To compare IC50 and MIC between phenotypes, we extracted day 5 data from cultures treated with 13 stepwise diluted concentrations of RIF and INH and plotted both IC50 and MIC values in figures 5 and 6 . The results obtained with this analysis of the RIF data revealed the cording phenotype as slightly more susceptible to the treatment than the planktonic, with both IC50 and MIC values (Table 1, Fig. 5A, Fig. 6A-B) being twice as high. Similarly, cording bacteria showed a higher INH-susceptibility over planktonic when comparing IC50 values (Table 1, Fig. 5B), while the MIC values of both was very similar (Table 1, Fig. 6C-D). Both IC50 and MIC values for all antibiotics tested followed the same trend with the cording phenotype being more susceptible compared to the planktonic (Table 1). After transposition of MIC values to nearest highest MIC according to ISO standard (Table 1), the difference between planktonic and cording phenotypes faded. Finally, in order to evaluate possible additive and synergistic effects 
Table 1. IC50 and MIC values for antibiotics.

\begin{tabular}{lllll} 
Antibiotic & IC50 & & \multicolumn{2}{l}{ MIC/Standard MIC } \\
\hline & planktonic & cording & planktonic & cording \\
\hline \hline Rifampicin & 0.0013 & 0.00070 & $0.017 / 0.016$ & $0.0088 / 0,016$ \\
Isoniazid & 0.00074 & 0.00036 & $0.0021 / 0,004$ & $0.0028 / 0,004$ \\
Linezolid & 0.36 & 0.15 & $1.7 * / 2$ & $1.1 * / 1$ \\
Levofloxacin & 0.29 & 0.23 & $1.1 * / 1$ & $0.91 * / 1$ \\
Ethambutol & 0.97 & 0.77 & $8.0 * / 8$ & $6.4 * / 8$ \\
Clofazimine & $0.28^{*}$ & $0.081^{*}$ & $0.31 / 0,5$ & $\mathrm{ND}$ \\
Moxifloxacin & $0,019^{*}$ & $0,0037^{*}$ & $\mathrm{ND}$ & $\mathrm{ND}$ \\
Pretomanid & $0,073^{*}$ & $0,037^{*}$ & $\mathrm{ND}$ & $\mathrm{ND}$
\end{tabular}

of antibiotics on the studied phenotypes, we performed a checkerboard assay with antibiotics at sub-inhibitory concentrations (concentrations summarized in Table 2). The experiment revealed that the cording phenotype was more susceptible to the combination of rifampicin, linezolid and pretomanid with several other drugs as compared to the planktonic bacteria (Fig. 7).

*not enough data to detect one or both limits of confidential interval $(95 \%)$

**MIC value transposed to nearest higher one according to the ISO standard

ND no value detected

Fig. 5. Dose response to antibiotics. Total area of aggregates in wells treated with antibiotics normalized to median of untreated controls $(\mathrm{N}=33)$ were used to calculate IC50 values. Black dotted line represents IC50 value for planktonic and red dotted line IC50 value for cording phenotype as determined by nonlinear regression (inhibitor vs response) with 4 parameters (A, 
199 cross X-axes at the point representing IC50 value for planktonic (black line) and cording (red 200 line) models.

A

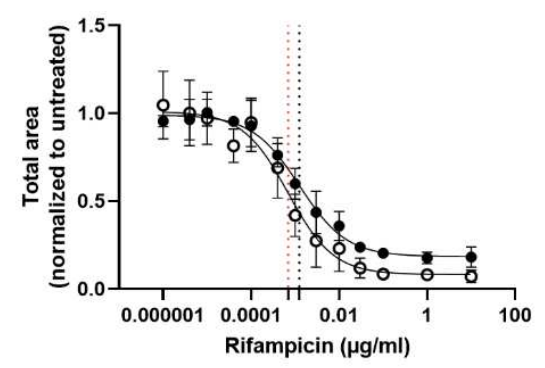

C

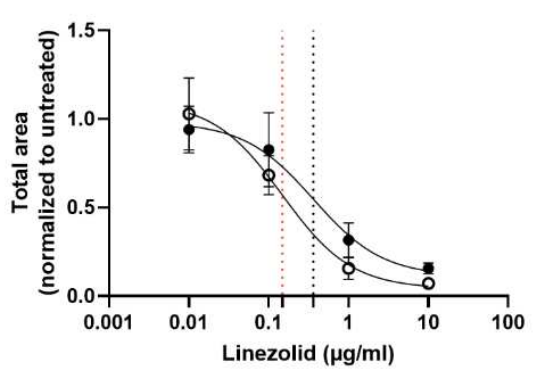

E

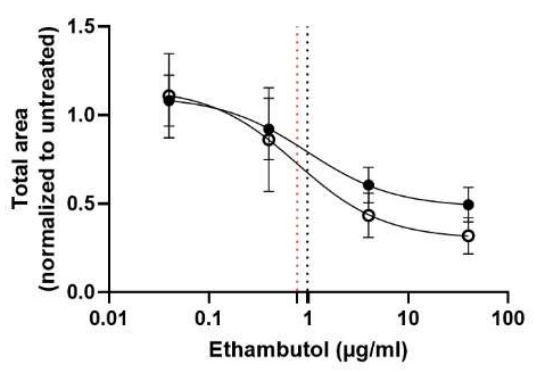

G

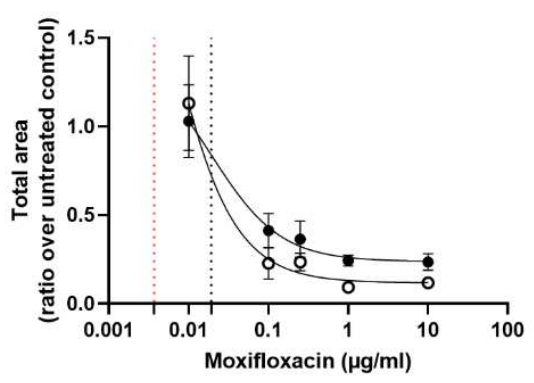

B

- planktonic

- cording

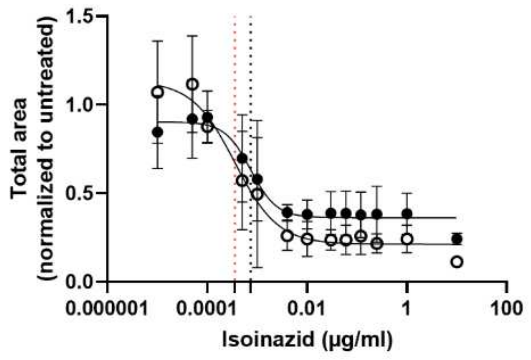

D

- planktonic

o- cording

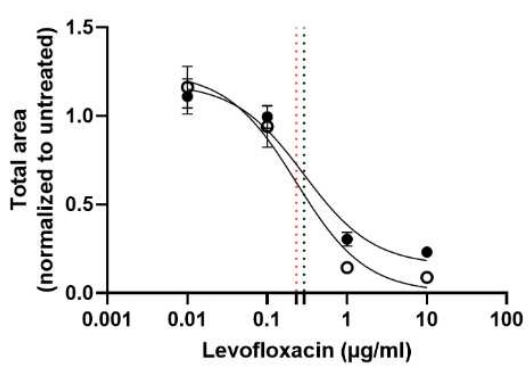

F

- planktonic

- cording

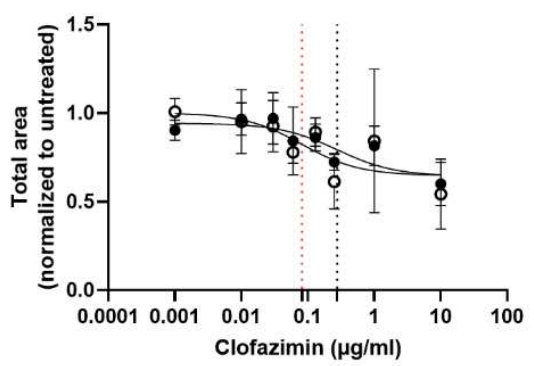

H

- planktonic

- cording

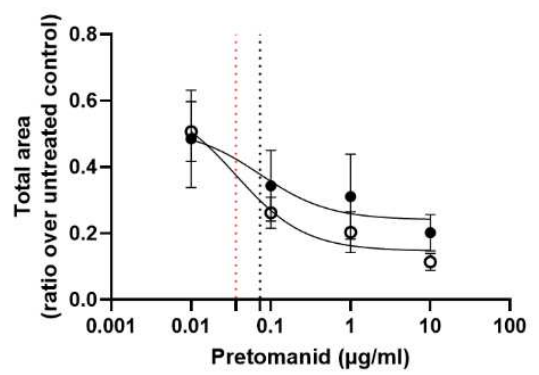

- planktonic

- cording

- planktonic

o- cording

- planktonic

- cording

- planktonic

- cording 
Fig. 6. MIC values for rifampicin (RIF) and isoniazid (INH). Gompertz functions was used to calculate MIC values at day5 based on total area of aggregates normalized to median of untreated controls ( $\mathrm{N}=33$ ) of the $\mathrm{A}), \mathrm{C})$ planktonic and $\mathrm{B}), \mathrm{D})$ cording model.

A

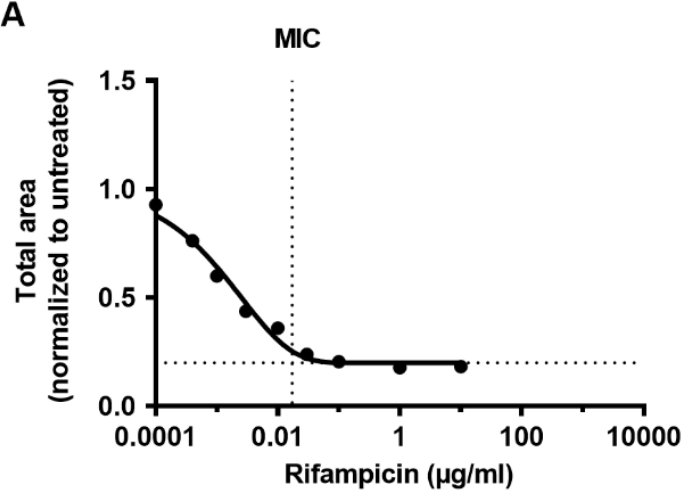

C

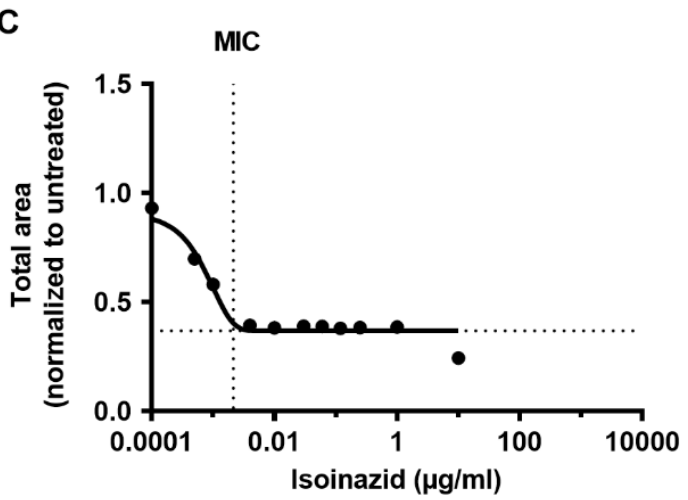

B

B MIC

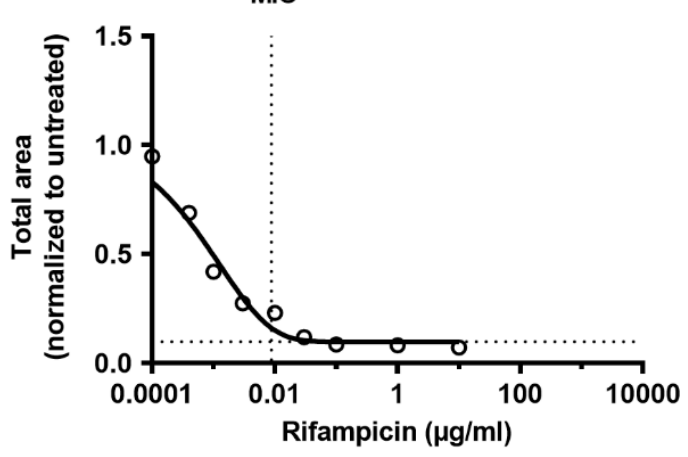

D

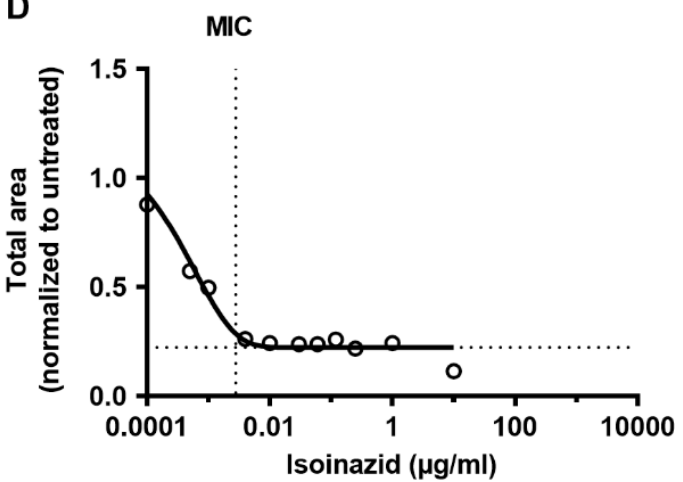


Fig. 7. Inhibition of $\mathbf{R v}$ growth by combination of antibiotics. $H 37 R v$ were treated by $(\mathrm{N}=33)$ were used in analysis. Data are presented as mean $\pm \mathrm{SD}(\mathrm{N}=3)$.
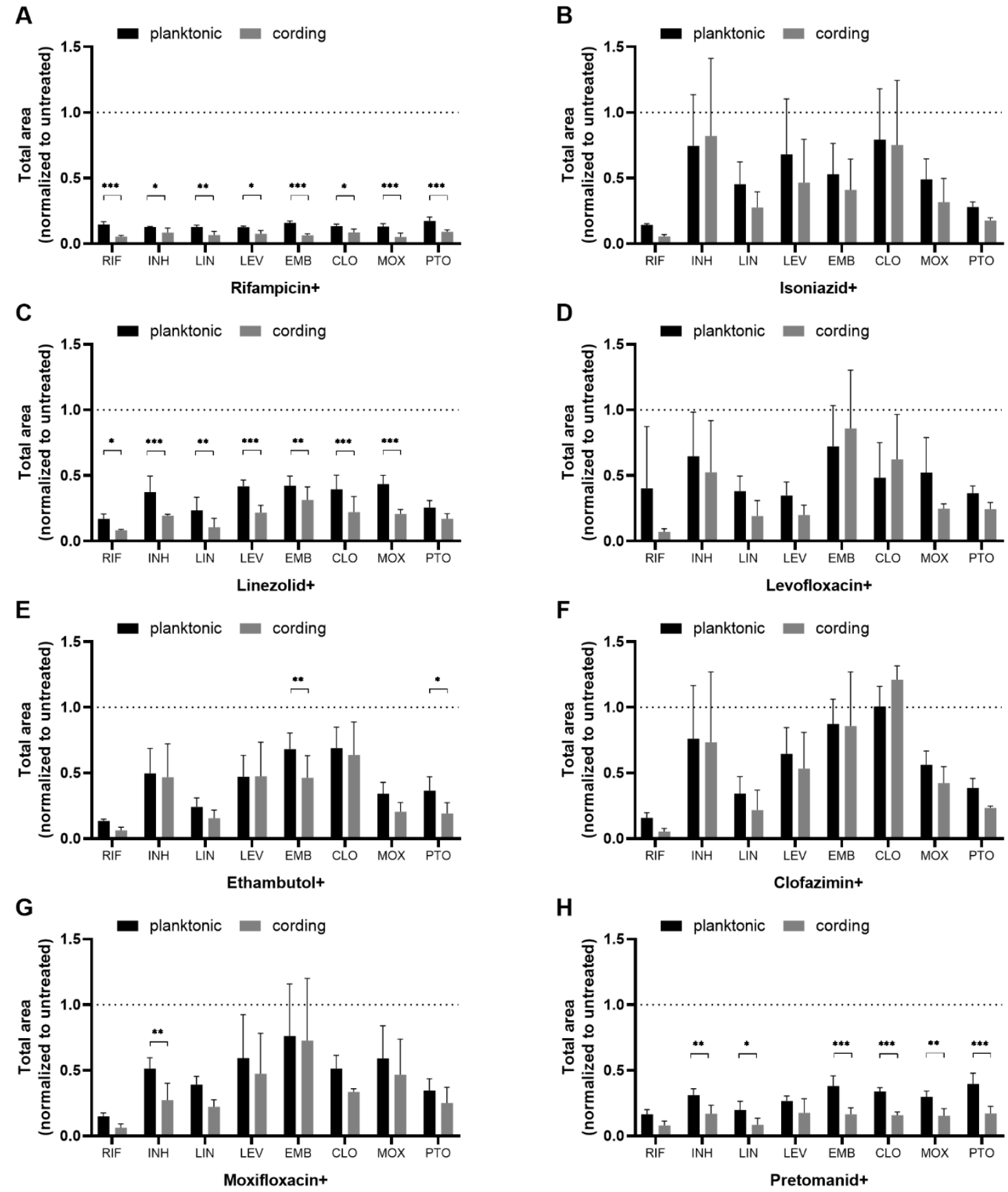
Our major goal in this study was to evaluate possibility to use automated live-cell imaging

222

223 system Incucyte S3 for high-trough put screening of assessment of TB drug susceptibility and compound libraries. We included two distinct phenotypes of Mtb in our high-content screening assay and investigated the potential differences in their antibiotic susceptibility. We could detect differences between the phenotypes as change of frequency in sizes of mycobacterial aggregates and monitor their growth and response to antibiotics. We were also able to calculate IC50 and MIC values for RIF and INH and the other antibiotics tested.

Heterogeneity of mycobacterial phenotypes in infected tissues and changing microenvironment during the course of TB poses a challenge for successful treatment that eliminates all forms of the pathogen. As we and others have previously demonstrated, the cording phenotype has possible bearings on TB severity, as it contributes to the pathophysiology $(7,8)$ and causes MET formation in infected macrophages (5). This prompted us to compare the effect of antibiotics on cording and planktonic mycobacteria.

Bacterial suspension is routinely homogenized as prerequisite for assessment of its concentration with help of OD measurements $(19,20)$. Although the generation of planktonic and cording bacteria occurred in broth, the phenotypes were keeping their distinct character in the cell culture medium we used afterwards. Even if the main niche of Mtb is the intracellular environment, mycobacteria in general often prevail extracellularly as cords $(21,22)$ and we reasoned that DMEM could better reflect such situations than enriched bacterial growth medium.

Detergents as Tween-80 in mycobacterial cultures ensures the dispersion of cells, promoting a homogenous culture, but the presence of detergent causes an artificial condition that can affect the growth characteristics and therefore also antibiotic susceptibility. It has been reported for 
244 bedaquiline, that antibiotic susceptibility, measured as MIC, changes with Tween-80 245 concentration (23) since detergent might interact with the antibiotic or even penetrate 246 mycobacterial cell walls as reviewed by Leisching et al (24). Phagocyte internalization of Mtb and the subsequent innate immune response can be affected by the presence of detergent in the 248 culture medium (25). Even if results of assays with planktonic, ideally dispersed, bacterial cells 249 can certainly be more reproducible in phenotypic drug-screening assays (26), they will not inform the development of drugs with optimal in vivo efficacy. Even with aggregating bacteria grown without detergent, we found variability of measurements in our experiments (less than $20 \%$ ) acceptable for the purpose of the assay.

The recorded data enabled us to reliably determine IC50 and MIC values for RIF and INH. Even though MICs were not determined in a standard method and using slightly different definitions than in clinical practice, they were in the range of clinical isolates in BACTEC 960 MGIT for RIF (0.016-0.125 mg/L) but slightly lower for INH (0.03-0.064 mg/L) $(27,28)$. Even if too few concentrations were used for the other tested antibiotics to determine IC50 and MIC values with sufficient certainty, they all seemed to follow similar trend with lower values for cording phenotype comparing to planktonic. The difference in antibiotic susceptibility between both phenotypes estimated by mathematical approximation disappeared after the MIC values were transposed to their ISO standards indicating that it was a marginal difference and should be interpreted with caution and more experimental evidence including larger range of tested concentrations would be needed to confirm that. It has been shown that cords consist of bacterial cells with smaller volume than cells in non-cording aggregates which can indicate more active replication within the cord (8). In line with this, the total area of cording bacteria was increasing previously (5). 


\section{Conclusion}

\section{Methods} MIC values.

We validated the live-cell imaging system Incucyte S3 for high-troughput screening of the growth and antibiotic susceptibility of two distinct phenotypes of Mtb. We were able to follow their growth dynamics and measure important parameters of drug activity such as IC50 and

\section{Bacterial culture}

H37Rv (American Type Culture Collection, ATCC 27294) harboring the pFPV2- plasmid encoding the green fluorescent protein (GFP) was grown and prepared as previously described (5). In short, the bacteria were grown for $2-3$ weeks at $37^{\circ} \mathrm{C}$ in Middlebrook $7 \mathrm{H} 9$ medium (BD Biosciences, USA) supplemented with $0.05 \%$ Tween-80 and albumin-dextrose-catalase enrichment (ADC, Becton Dickinson) using $20 \mu \mathrm{g} / \mathrm{ml}$ of kanamycin (Sigma-Aldrich, MO) as a selective antibiotic. The bacteria were reseeded as standing and shaken cultures before the experiment. The standing culture was passaged in a new medium with $0.05 \%$ Tween-80 and incubated at $37^{\circ} \mathrm{C}$ for additional 5 days while the shaken culture was made in medium without Tween-80 and put on a shaker at $260 \mathrm{rpm} 3$ days prior to the experiment. 
The bacterial suspensions from both tubes (i.e.shaken and standing) were prepared as described earlier (29). Briefly, the bacterial suspensions were centrifuged twice at 5,000 x g for $5 \mathrm{~min}$ in phosphate-buffered saline (PBS) supplemented with $0.05 \%$ Tween-80 and passaged through a sterile syringe equipped with 27-gauge needle to remove bacterial aggregates. After the final wash, bacterial pellets from both tubes were resuspended in antibiotic-free DMEM (Gibco) containing $25 \mathrm{mM}$ HEPES (Gibco), $2 \mathrm{mM}$ L-glutamine (Gibco) and 10\% active human serum (pooled from 5 healthy donors, blood bank of Linköping University Hospital) (ABF medium) and the concentration as CFU/ml was determined by measuring optical density (OD 600$)$. Since ABF medium is commonly used in our lab in models of Mtb growing intracellularly in human cells and supports bacterial growth very effectively, we decided to continue cultivation in it after phenotypes have been generated in broth. Finally, $35 \mu \mathrm{l}$ of each bacterial suspension was seeded in separate 384-well black clear-bottom plates (BD, Falcon) and placed in Incucyte S3 (IncuCyte ${ }^{\circledR}$ Live-Cell Analysis System, Sartorius) for live cell imaging at $37^{\circ} \mathrm{C}$ for 48 hours to allow initiation of growth in the DMEM. Images (2/well) at 20x magnification were captured with $2 \mathrm{~h}$ intervals. Selected first and second-line antibiotics available for TB treatment were dissolved either in sterile, deionized water or $100 \%$ dimethyl sulfoxide (DMSO) to obtain stock solutions and then diluted in $\mathrm{ABF}$ medium to achieve the final required concentrations (summarized in Table 2). $35 \mu 1$ of 2 times the final concentration of antibiotic solutions was added in respective wells after 48 hours of addition of bacterial suspension to plate to make the final volume of $70 \mu$ l. The plate was then placed in Incucyte $S 3$ for additional 5 days (Fig. 1). The experiment layout was designed such as one well was used for each antibiotic treatment and 33 wells were left for untreated controls on each plate and three replicated experiments were performed. Planktonic and cording bacteria were always seeded on separate plates. We performed also control experiment when $\mathrm{H} 37 \mathrm{Rv}$ was growing as standing culture at $7 \mathrm{H} 9$ 
316 filtered through $0,5 \mu \mathrm{m}$ filter and seeded on 96-well plate in fresh medium with or without

317 Tween-80.

318 Fig. 1. Scheme of experimental layout

$$
\begin{gathered}
\text { bacterial culture } \\
\text { started }
\end{gathered} \quad \text { 2-3 weeks }\left[\begin{array}{c}
\text { planktonic } \\
\text { standing with Tween for } 5 \text { days } \\
\text { cording } \\
\text { shaken without Tween for } 3 \text { days }
\end{array}\right.
$$

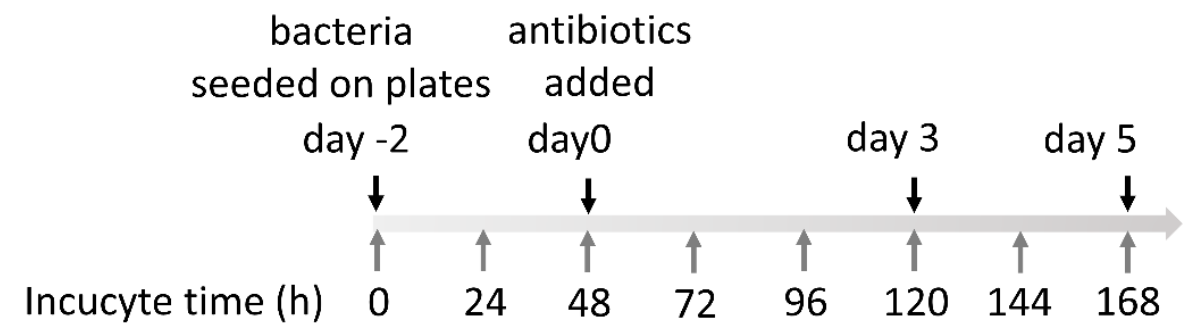

319

Table 2. Solvent used in preparation of antibiotics and concentrations used in assays.

\begin{tabular}{llll}
\hline Antibiotic & Solvent & $\begin{array}{l}\text { Dose response } \\
\text { ranges }(\mu \mathrm{g} / \mathrm{ml})\end{array}$ & $\begin{array}{l}\text { Checkerboard } \\
\text { assay* }(\mu \mathrm{g} / \mathrm{ml})\end{array}$ \\
\hline \hline Rifampicin & DMSO & $10-1 \times 10^{-6}$ & 1 \\
Isoniazid & $\mathrm{H}_{2} \mathrm{O}$ & $10-1 \times 10^{-5}$ & 0.1 \\
Linezolid & DMSO & $10-0.01$ & 1 \\
Levofloxacin & DMSO & $10-0.01$ & 1 \\
Ethambutol & DMSO & $40-0.04$ & 4 \\
Clofazimine & DMSO & $10-0.001$ & 10 \\
Moxifloxacin & $\mathrm{H}_{2} \mathrm{O}$ & $10-0.01$ & 0.25 \\
Pretomanid & DMSO & $10-0.01$ & 1
\end{tabular}

$321 *$ subinhibitory concentrations 
Fluorescent objects in both planktonic and cording model were identified with the help of inbuild IncuCyte S3 software enabling segmentation and background correction. Data on total area $\left(\mu \mathrm{m}^{2}\right)$ of identified fluorescent objects were collected and exported from the Incucyte S3 into Excel and GraphPad (version 8.4.3.) for summarizing the results and statistical analysis. Intra- and inter-assay variability was calculated as previously published (19). Variability of total area measurements within each plate (intra-assay variability) was calculated based on replicated $(\mathrm{N}=33)$ samples of untreated $\mathrm{Rv}$ after 7 days of growth. Variability of measurements between platesinter-assay variability) was based on total area measurements of untreated $\mathrm{Rv}$ at three separate experiments (first well of 33 replicated was chosen for each experiment) at the same incubation time. Both values were expressed as the coefficient of variation (CV\%). Twoway RM ANOVA with Sidaks's multiple comparison test was used to compare differences in total area of fluorescent objects between untreated planktonic and cording bacteria. Total area of objects after antibiotic treatment was normalized to the median of total area of all untreated controls in each experiment (Additional files 3-6) and mean of three repetitive experiments with standard deviation in error bars were than used in further analysis.

\section{Analysis of frequency of object sizes}

Data on frequency of sizes of fluorescent objects in untreated control wells in both models were extracted from Incucyte software. Size intervals were chosen so they were logarithmically distributed up to $10^{4} \mu \mathrm{m}^{2}$. Since some measurements had no objects present, those points were filtered out and median of replicated wells was used in further analysis. Difference between untreated controls in planktonic and cording model was than analysed by multiple t-tests with 
correction for multiple comparisons using Holm-Sidak method in GraphPad (version 8.4.3.) . Data on frequency of object sizes in wells treated with antibiotics were after filtering out the measurements without present objects analysed directly since there was only one well per treatment in each experiment. Changes in distribution of objects sizes during the treatment of bacteria with RIF and INH were analysed with multiple t-tests as mentioned for untreated controls.

\section{Analysis of dose response to antibiotics and MIC values}

As recommended by GraphPad, IC50 values were calculated by fitting data into dose reponse curves (inhibitor vs response) by nonlinear regression with three parameters and standard slope for those antibiotics where only few concentrations were tested. Nonlinear regression with four parameters and variable slope was used in case of RIF and INH, where 13 concentrations were avalaible. MIC defined as lowest concentration enough to effectively reduce the growth of bacteria relatively to control was determined in GraphPad using modified Gompertz function (30). The MIC was then transposed to nearest higher MIC value using the the log2-scale according to ISO-standard.

\section{Image acquisition, processing and statistical analysis of largest aggregates}

To measure the growth of a single aggregate, images were analyzed through MATLAB image processing with in-house scripts (scripts will be available upon request). Images were extracted from large aggregate identified in well without antibiotics (Additional file 8: Movie S1). The 
377 Not applicable

378 Availability of data and materials

379 The datasets used and/or analysed during the current study are available from the corresponding 380 author on reasonable request.

381 Competing interests

382 The authors declare that they have no competing interests.

$383 \quad$ Funding

$$
\Delta \mathrm{A}=\mathrm{A}_{\mathrm{i}+1}-\mathrm{A}_{\mathrm{i}} ; \text { and } \Delta \mathrm{t}=\mathrm{t}_{\mathrm{i}+1}-\mathrm{t}_{\mathrm{i}} .
$$

where $\Delta \mathrm{A}$ is the change of area; $\Delta \mathrm{t}$ is the change of time, and $\mathrm{i}$ is the unit vector.

\section{Declarations}

\section{Acknowledgements}

Ethics approval and consent to participate

Not applicable

\section{Consent for publication}

Funding 
SK and BA (shared first authorship): Data acquisition, data analysis, writing and revision of the manuscript, JD: Data analysis, writing and revision of the manuscript, TS: Supervision of the study and revision of the manuscript. ML: Supervision of the study and revision of the manuscript.

1. Adeniji AA, Knoll KE, Loots DT. Potential anti-TB investigational compounds and drugs with repurposing potential in TB therapy: a conspectus. Appl Microbiol Biotechnol. 2020;104(13):5633-62.

2. Middlebrook G, Dubos RJ, Pierce C. Virulence and Morphological Characteristics of Mammalian Tubercle Bacilli. J Exp Med. 1947;86(2):175-84.

$3 . \quad$ Hall-Stoodley L, Brun OS, Polshyna G, Barker LP. Mycobacterium marinum biofilm formation reveals cording morphology. FEMS Microbiol Lett. 2006;257(1):43-9.

4. Julian E, Roldan M, Sanchez-Chardi A, Astola O, Agusti G, Luquin M. Microscopic cords, a virulence-related characteristic of Mycobacterium tuberculosis, are also present in nonpathogenic mycobacteria. J Bacteriol. 2010;192(7):1751-60.

5. Kalsum S, Braian C, Koeken V, Raffetseder J, Lindroth M, van Crevel R, et al. The Cording Phenotype of Mycobacterium tuberculosis Induces the Formation of Extracellular Traps in Human Macrophages. Front Cell Infect Microbiol. 2017;7:278.

6. $\quad$ Arias L, Cardona P, Catala M, Campo-Perez V, Prats C, Vilaplana C, et al. Cording Mycobacterium tuberculosis Bacilli Have a Key Role in the Progression towards Active Tuberculosis, Which is Stopped by Previous Immune Response. Microorganisms. 2020;8(2).

7. Ufimtseva EG, Eremeeva NI, Petrunina EM, Umpeleva TV, Bayborodin SI, Vakhrusheva DV, et al. Mycobacterium tuberculosis cording in alveolar macrophages of patients with pulmonary tuberculosis is likely associated with increased mycobacterial virulence. Tuberculosis (Edinb). 2018;112:1-10.

8. Lerner TR, Queval CJ, Lai RP, Russell MR, Fearns A, Greenwood DJ, et al. Mycobacterium tuberculosis cords within lymphatic endothelial cells to evade host immunity. JCI Insight. 2020;5(10).

9. Manjunatha UH, Smith PW. Perspective: Challenges and opportunities in TB drug discovery from phenotypic screening. Bioorg Med Chem. 2015;23(16):5087-97.

10. Grzelak EM, Choules MP, Gao W, Cai G, Wan B, Wang Y, et al. Strategies in antiMycobacterium tuberculosis drug discovery based on phenotypic screening. J Antibiot (Tokyo). 2019;72(10):719-28.

11. Andries K, Verhasselt P, Guillemont J, Gohlmann HW, Neefs JM, Winkler H, et al. A diarylquinoline drug active on the ATP synthase of Mycobacterium tuberculosis. Science. 2005;307(5707):223-7. 

antituberculosis drug activity in vitro and in macrophages using bioluminescence. J Antimicrob Chemother. 2012;67(2):404-14.

13. Katzir I, Cokol M, Aldridge BB, Alon U. Prediction of ultra-high-order antibiotic combinations based on pairwise interactions. PLoS Comput Biol. 2019;15(1):e1006774.

14. Song OR, Deboosere N, Delorme V, Queval CJ, Deloison G, Werkmeister E, et al. Phenotypic assays for Mycobacterium tuberculosis infection. Cytometry A. 2017;91(10):983-94. 15. Stanley SA, Barczak AK, Silvis MR, Luo SS, Sogi K, Vokes M, et al. Identification of hosttargeted small molecules that restrict intracellular Mycobacterium tuberculosis growth. PLoS Pathog. 2014;10(2):e1003946.

16. Christophe T, Jackson M, Jeon HK, Fenistein D, Contreras-Dominguez M, Kim J, et al. High content screening identifies decaprenyl-phosphoribose 2 ' epimerase as a target for intracellular antimycobacterial inhibitors. PLoS Pathog. 2009;5(10):e1000645.

17. Manning AJ, Ovechkina Y, McGillivray A, Flint L, Roberts DM, Parish T. A high content microscopy assay to determine drug activity against intracellular Mycobacterium tuberculosis. Methods. 2017;127:3-11.

18. Queval CJ, Song OR, Delorme V, lantomasi R, Veyron-Churlet R, Deboosere N, et al. A microscopic phenotypic assay for the quantification of intracellular mycobacteria adapted for highthroughput/high-content screening. J Vis Exp. 2014(83):e51114.

19. Eklund D, Welin A, Schon T, Stendahl O, Huygen K, Lerm M. Validation of a mediumthroughput method for evaluation of intracellular growth of Mycobacterium tuberculosis. Clin Vaccine Immunol. 2010;17(4):513-7.

20. Welin A, Raffetseder J, Eklund D, Stendahl O, Lerm M. Importance of phagosomal functionality for growth restriction of Mycobacterium tuberculosis in primary human macrophages. J Innate Immun. 2011;3(5):508-18.

21. Bernut A, Herrmann JL, Kissa K, Dubremetz JF, Gaillard JL, Lutfalla G, et al. Mycobacterium abscessus cording prevents phagocytosis and promotes abscess formation. Proc Natl Acad Sci U S A. 2014;111(10):E943-52.

22. Johansen MD, Kremer L. Large Extracellular Cord Formation in a Zebrafish Model of Mycobacterium kansasii Infection. J Infect Dis. 2020;222(6):1046-50.

23. Lounis N, Vranckx L, Gevers T, Kaniga K, Andries K. In vitro culture conditions affecting minimal inhibitory concentration of bedaquiline against $M$. tuberculosis. Med Mal Infect. 2016;46(4):220-5.

24. Leisching G, Pietersen RD, Wiid I, Baker B. Virulence, biochemistry, morphology and host-interacting properties of detergent-free cultured mycobacteria: An update. Tuberculosis (Edinb). 2016;100:53-60.

25. Leisching G, Pietersen RD, Mpongoshe V, van Heerden C, van Helden P, Wiid I, et al. The Host Response to a Clinical MDR Mycobacterial Strain Cultured in a Detergent-Free Environment: A Global Transcriptomics Approach. PLoS One. 2016;11(4):e0153079.

26. Cheng N, Porter MA, Frick LW, Nguyen Y, Hayden JD, Young EF, et al. Filtration improves the performance of a high-throughput screen for anti-mycobacterial compounds. PLoS One. 2014;9(5):e96348.

27. Svensson RJ, Niward K, Davies Forsman L, Bruchfeld J, Paues J, Eliasson E, et al. Individualised dosing algorithm and personalised treatment of high-dose rifampicin for tuberculosis. Br J Clin Pharmacol. 2019;85(10):2341-50.

28. Schon T, Jureen P, Giske CG, Chryssanthou E, Sturegard E, Werngren J, et al. Evaluation of wild-type MIC distributions as a tool for determination of clinical breakpoints for Mycobacterium tuberculosis. J Antimicrob Chemother. 2009;64(4):786-93.

29. Abuzeid N, Kalsum S, Koshy RJ, Larsson M, Glader M, Andersson H, et al. Antimycobacterial activity of selected medicinal plants traditionally used in Sudan to treat infectious diseases. J Ethnopharmacol. 2014;157:134-9. 

inhibitory concentration (MIC) and non-inhibitory concentration (NIC) values. J Appl Microbiol. 2000;88(5):784-90.

\section{Additional files}

\section{Additional file 1 Table S1.xlsx Frequency of aggregate sizes in untreated controls of} planktonic and cording bacteria. Data represents median number of 33 replicated wells of control untreated H37Rv.

Additional file 2 Fig S1.tif Growth of planktonic and cording bacteria. Growth was measured as a difference in total area as absolute value A) or ratio over d0 B). Day 0 refers to the time point when antibiotics were added after 2 days of pre-culture in the 96-well plate. Data are represented as mean $\pm \mathrm{SD}(\mathrm{N}=3)$ based on median value of 33 replicated wells in each experiment.

Additional file 3 Fig S2.tif Growth of planktonic bacteria exposed to rifampicin (RIF). Growth of aggregates at highest $(10 \mu \mathrm{g} / \mathrm{ml})$ and lowest $\left(1 \times 10^{-6} \mu \mathrm{g} / \mathrm{ml}\right)$ concentration of rifampicin based on differences in total growth as A), B) absolute values or C), D) normalized to median of all wells with untreated controls $(\mathrm{N}=33) \mathrm{B})$. D0 refers to the time point when antibiotics were added after 2 days of pre-culture in the 96-well plate. Data represents values for each of three experiments separately.

Additional file 4 Fig S3.tif Growth of cording bacteria exposed to rifampicin (RIF). Growth of aggregates at highest $(10 \mu \mathrm{g} / \mathrm{ml})$ and lowest $\left(1 \times 10^{-6} \mu \mathrm{g} / \mathrm{ml}\right)$ concentration of rifampicin based on differences in total growth as A), B) absolute values or C), D) normalized to median of all wells with untreated controls $(\mathrm{N}=33) \mathrm{B})$. D0 refers to the time point when antibiotics were added after 2 days of pre-culture in the 96-well plate. Data represents values for each of three experiments separately. 
Growth of aggregates at highest $(10 \mu \mathrm{g} / \mathrm{ml})$ and lowest $\left(1 \times 10^{-5} \mu \mathrm{g} / \mathrm{ml}\right)$ concentration of isoniazid

502

503

504

505

506

507

508

509

510

511

512

513

514

515

based on differences in total growth as A), B) absolute values or C), D) normalized to median of all wells with untreated controls $(\mathrm{N}=33) \mathrm{B})$. D0 refers to the time point when antibiotics were added after 2 days of pre-culture in the 96-well plate. Data represents values for each of three experiments separately.

Additional file 6 Fig S5.tif Growth of cording bacteria exposed to isoniazid (INH). Growth of aggregates at highest $(10 \mu \mathrm{g} / \mathrm{ml})$ and lowest $\left(1 \times 10^{-5} \mu \mathrm{g} / \mathrm{ml}\right)$ concentration of isoniazid based on differences in total growth as A), B) absolute values or C), D) normalized to median of all wells with untreated controls $(\mathrm{N}=33) \mathrm{B})$. D0 refers to the time point when antibiotics were added after 2 days of pre-culture in the 96-well plate. Data represents values for each of three experiments separately.

Additional file 7 Table S2.xlsx Intra- and inter-assay variability. Variability between growth measurements (total area as $\mu \mathrm{m}^{2}$ /image) within each plate and between plates presented as coefficient of variation $(\%)$.

Additional file 8 Movie S1.mp4 Growth of single aggregate. 
Figures

bacterial culture started planktonic

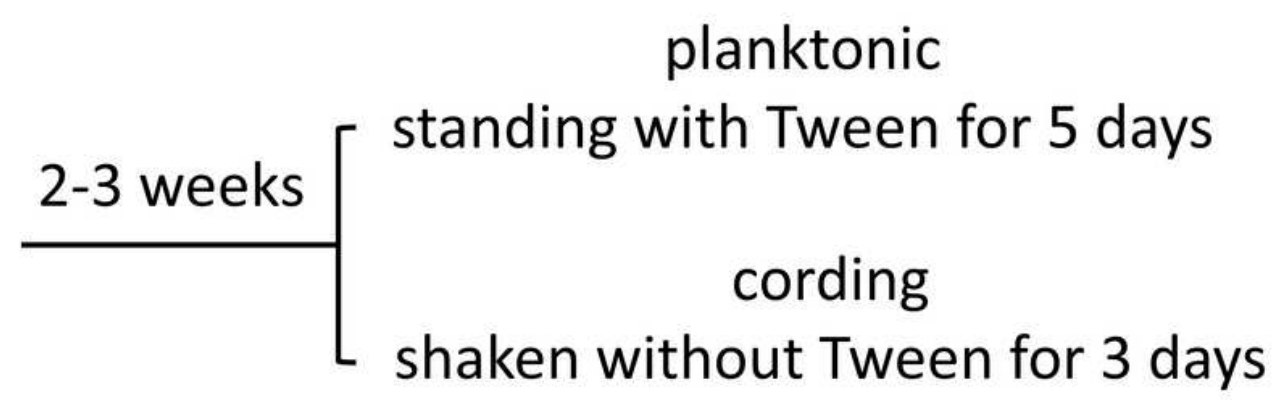

$\begin{array}{cc}\text { bacteria } & \text { antibiotics } \\ \text { seeded on plates } & \text { added }\end{array}$

day -2

Incucyte time (h) $\downarrow$

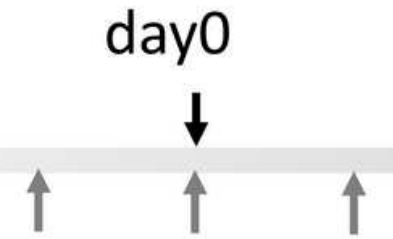

$0 \quad 24$

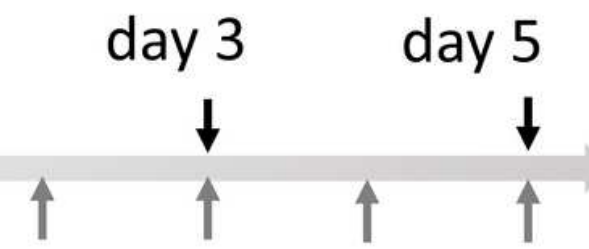

$\begin{array}{llll}96 & 120 & 144 & 168\end{array}$

Figure 1

Scheme of experimental layout 
A

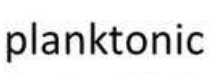
in DMEM

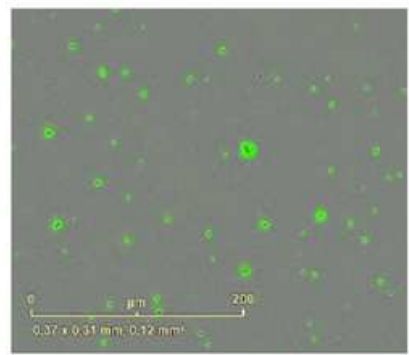

day-2

day0

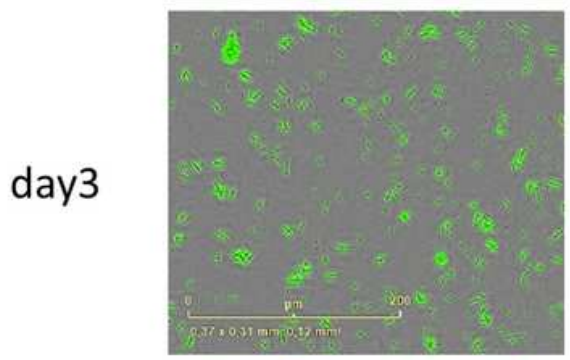

day5
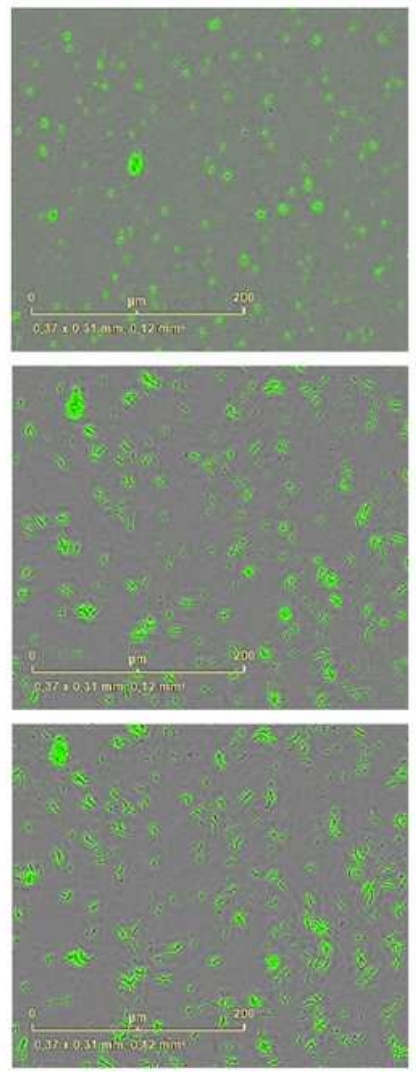

B cording in DMEM
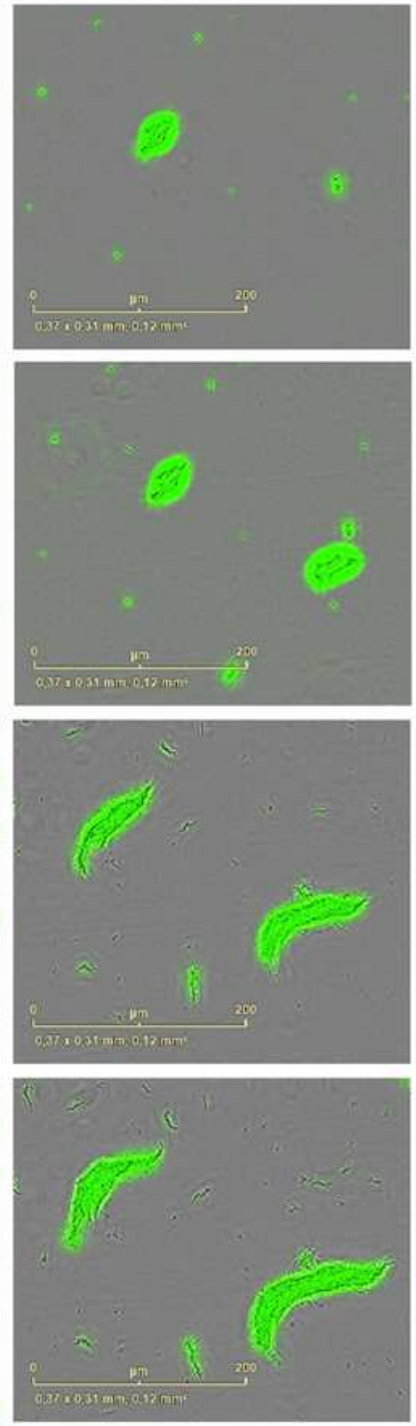

C

$\mathrm{Rv}$ in $7 \mathrm{H} 9$ with Tween-80

$\mathrm{Rv}$ in $7 \mathrm{H} 9$ without Tween-80
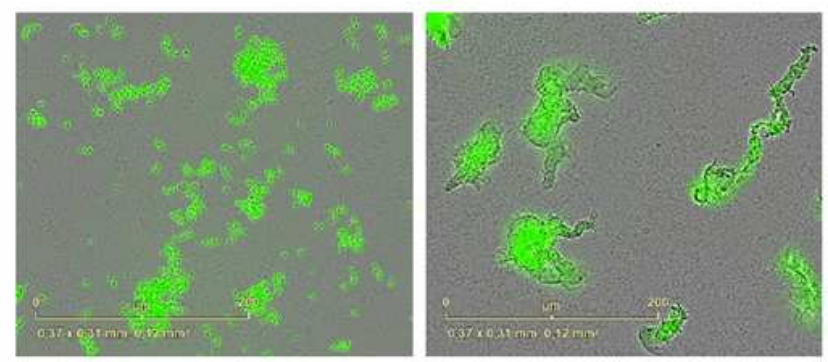

D

Day -2

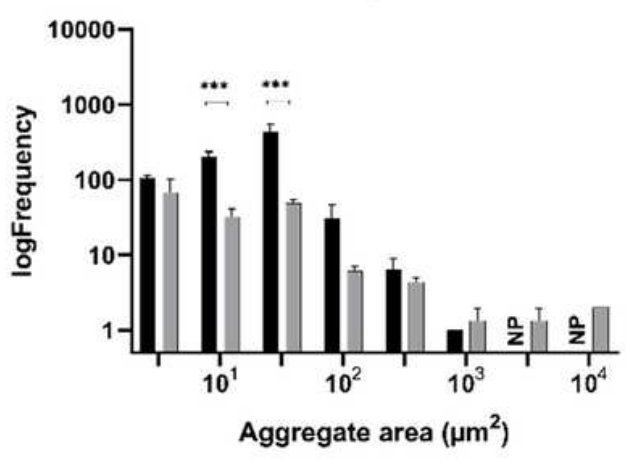

E

Day 5

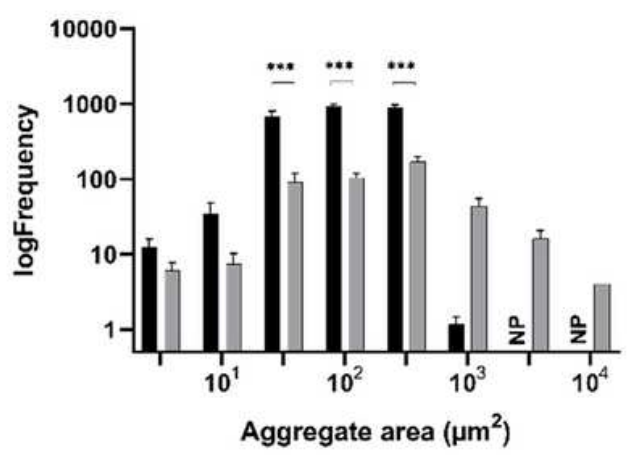

- planktonic ㅁarding

- planktonic cording

\section{Figure 2}

Morphological appearance and size of aggregates in planktonic and cording models. H37Rv growing in DMEM as planktonic (A) and cording (B) bacteria are shown at different time points. H37Rv grown in broth with or without Tween-80 (Tween) as indicated (C). Frequency plots of the distribution of aggregate sizes at day -2 (D) and day 5 (E). Columns represent size intervals and are logarithmically distributed up to $104 \mu \mathrm{m} 2$. NP (non-present) marks intervals where no objects were identified. Data are presented as mean $\pm S D(N=3)$. 
planktonic

A

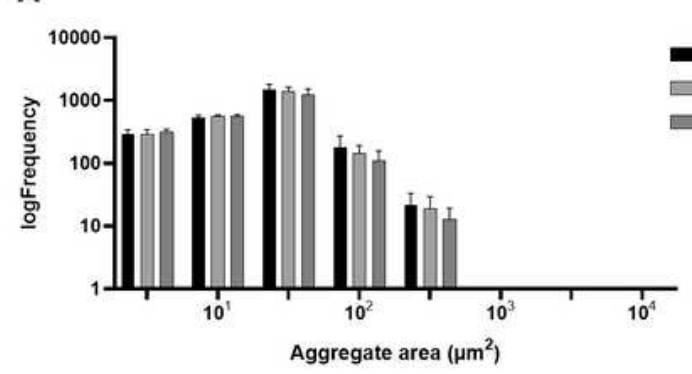

C RIF $1 \times 10^{-3} \mu \mathrm{g} / \mathrm{ml}$

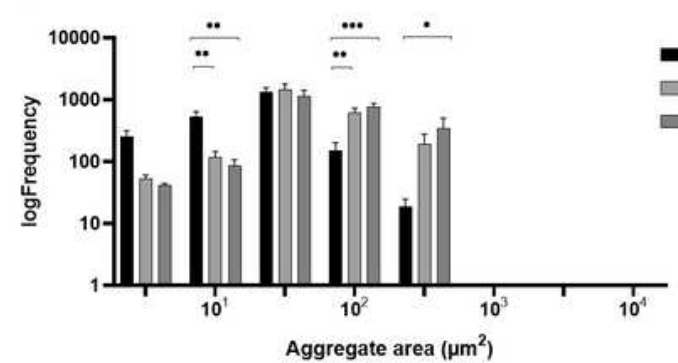

E

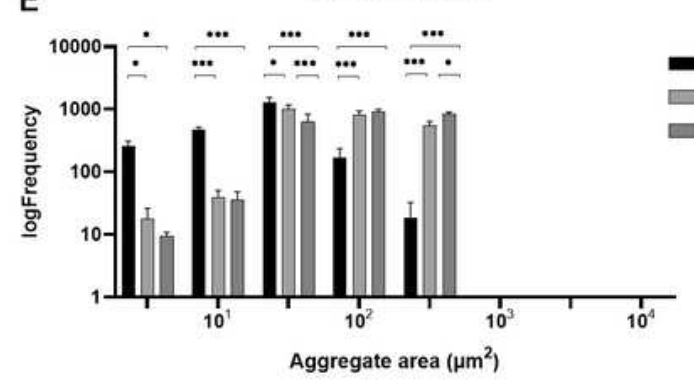

G

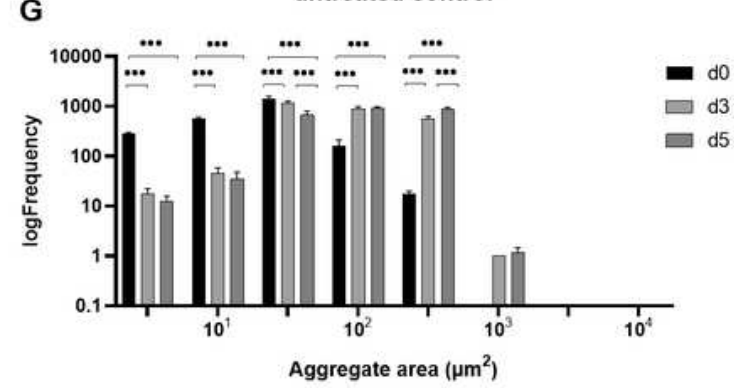

cording

B

RIF $10 \mu \mathrm{g} / \mathrm{ml}$

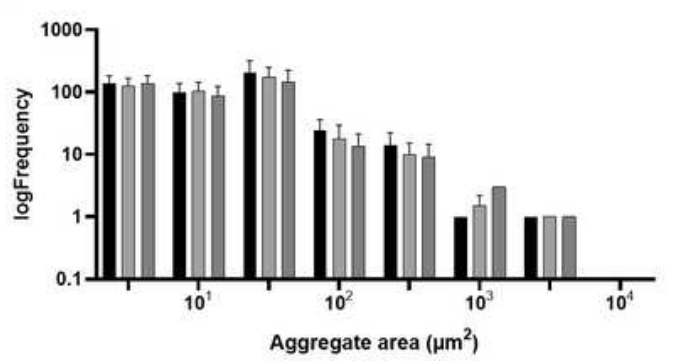

D RIF $1 \times 10^{-3} \mu \mathrm{g} / \mathrm{ml}$

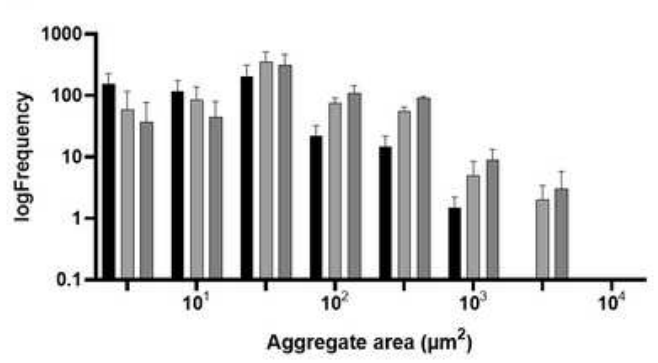

- do $\mathrm{d} 3$

- d5
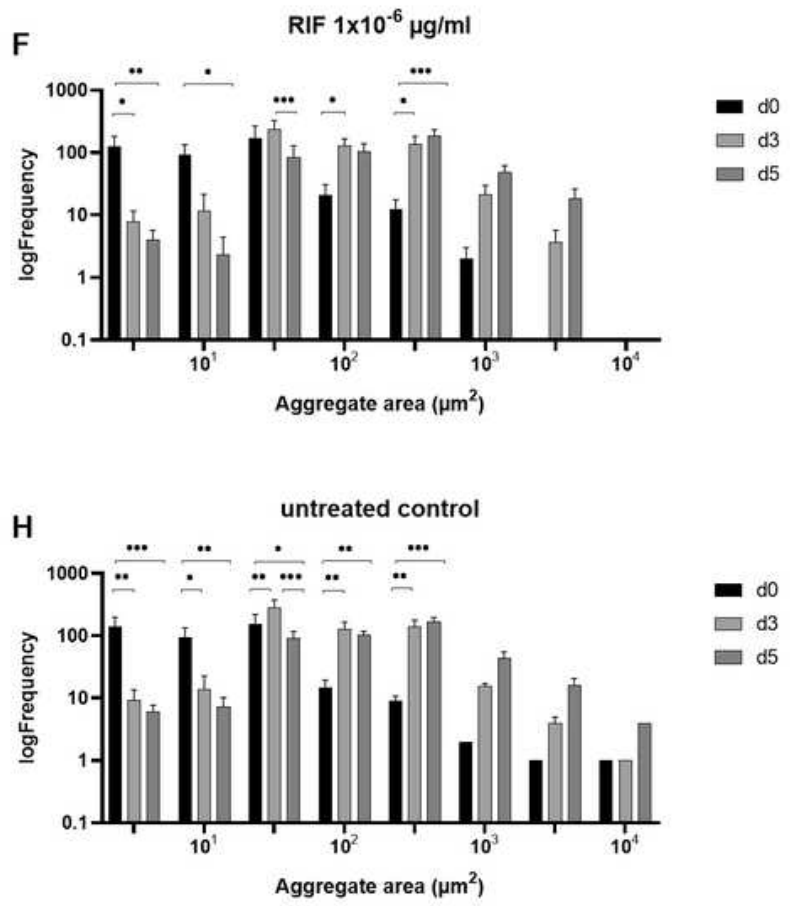

\section{Figure 3}

Frequency of aggregate sizes during bacterial growth and in response to rifampicin (RIF). A, C, E) Frequency plots summarizing data from planktonic phenotype and $B, D, F$ ) data from cording phenotype exposed to different concentrations of rifampicin after 3 and 5 days of incubation. $G, H$ ) Frequency plots of untreated controls for planktonic and cording phenotype respectively. Data are presented as mean \pm SD $(\mathrm{N}=3)$. 
planktonic

A

INH 10 $\mu \mathrm{g} / \mathrm{ml}$

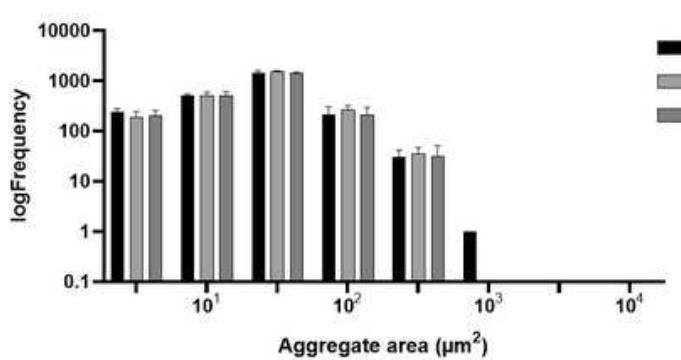

C

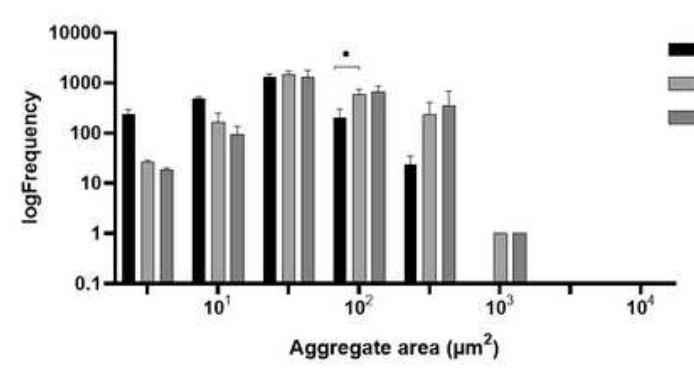

E

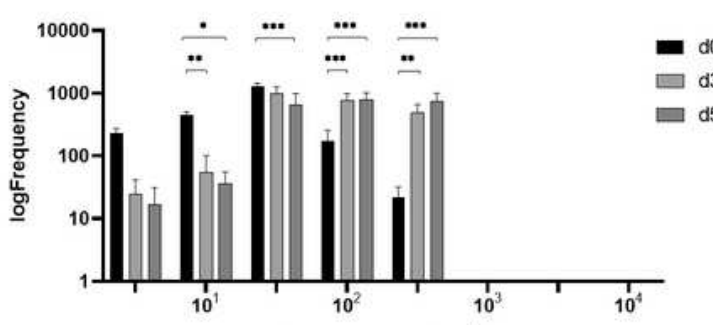

Aggregate area $\left(\mu \mathrm{m}^{2}\right)$

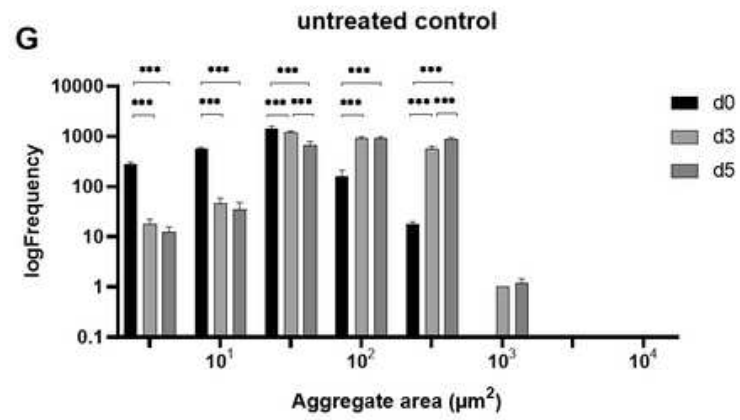

cording

B

INH 10 $\mu \mathrm{g} / \mathrm{ml}$

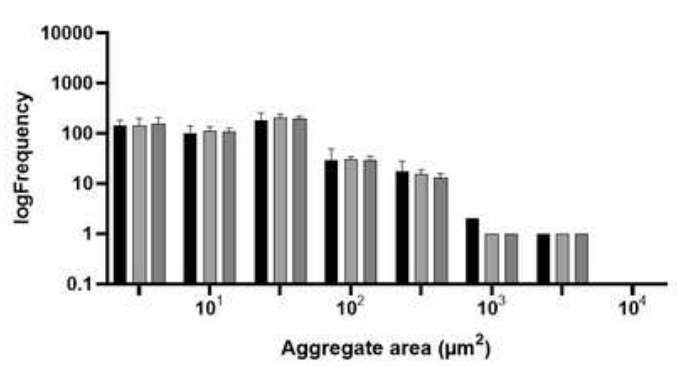

INH $1 \times 10^{-3} \mu \mathrm{g} / \mathrm{ml}$

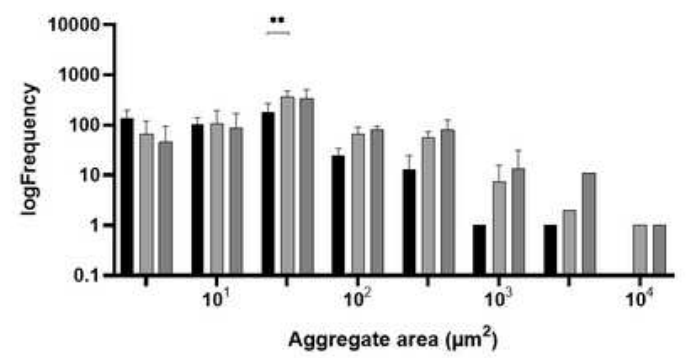

F

INH $1 \times 10^{-5} \mu \mathrm{g} / \mathrm{ml}$

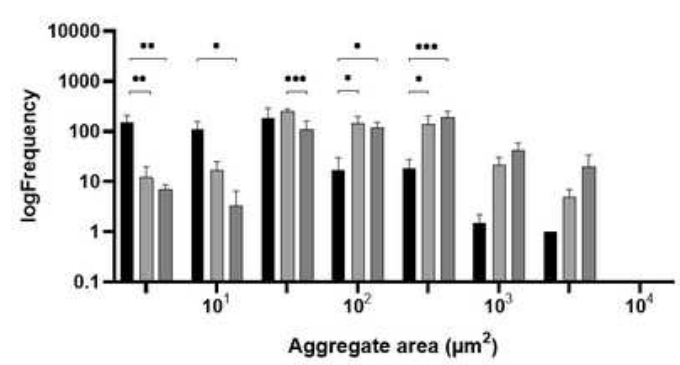

H

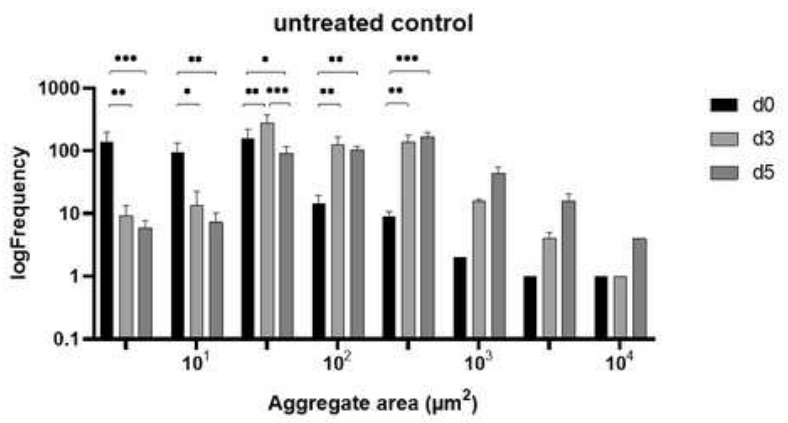

Figure 4

Frequency of aggregate sizes during bacterial growth and in response to isoniazid (INH). A, C, E) Frequency plots summarizing data from planktonic phenotype and $B, D, F$ ) data from cording phenotype exposed to different concentrations of isoniazid after 3 and 5 days of incubation. $G, H$ ) Frequency plots of untreated controls for planktonic and cording phenotype respectively. Data are presented as mean \pm SD $(\mathrm{N}=3)$. 
A

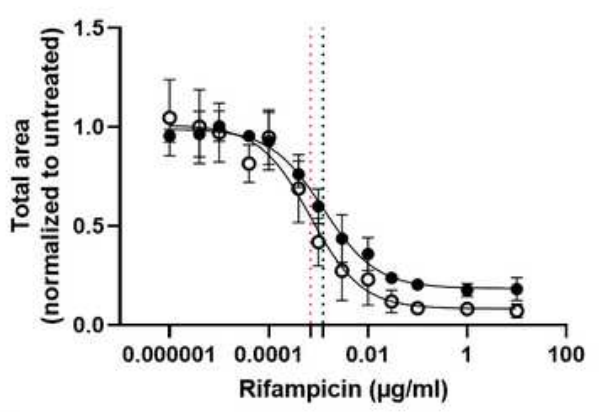

C

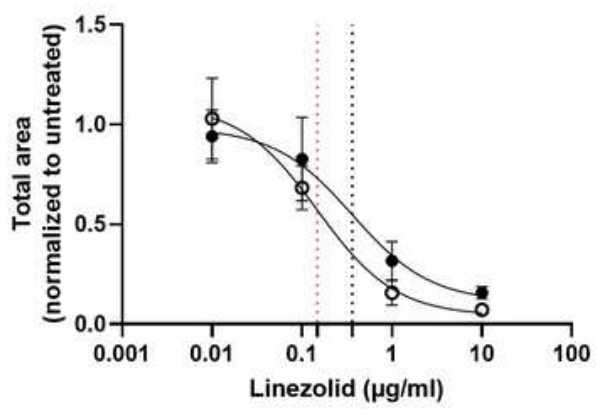

E

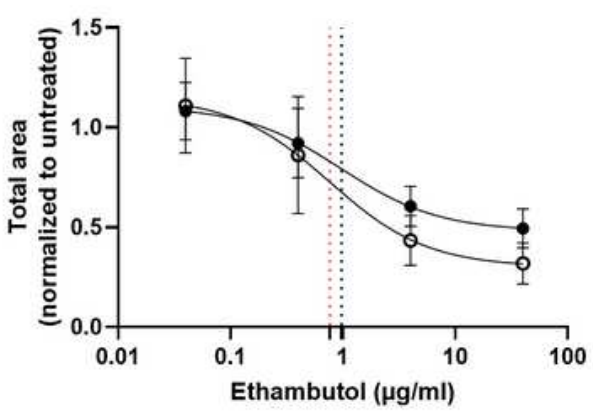

G

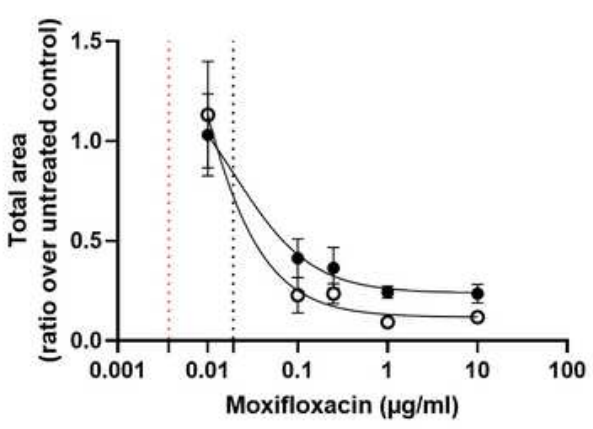

B

- planktonic

- cording

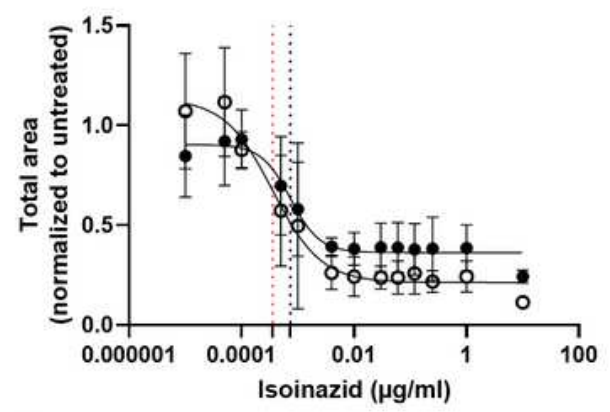

D

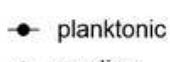

- cording

- planktonic

- cording

- planktonic

- cording

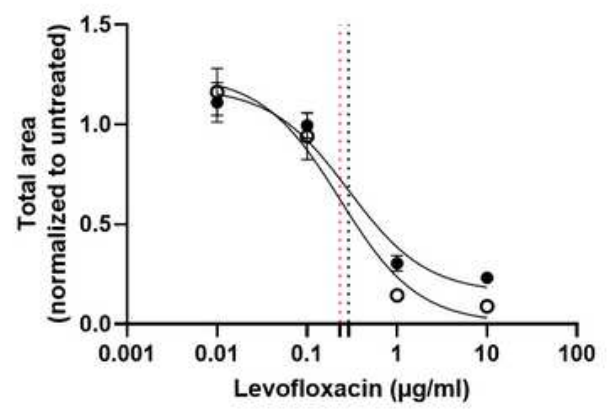

F

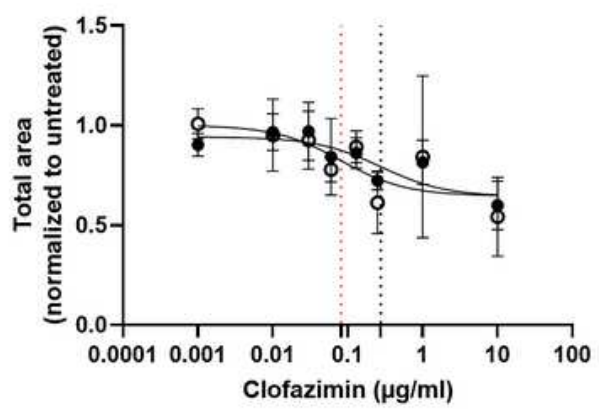

H

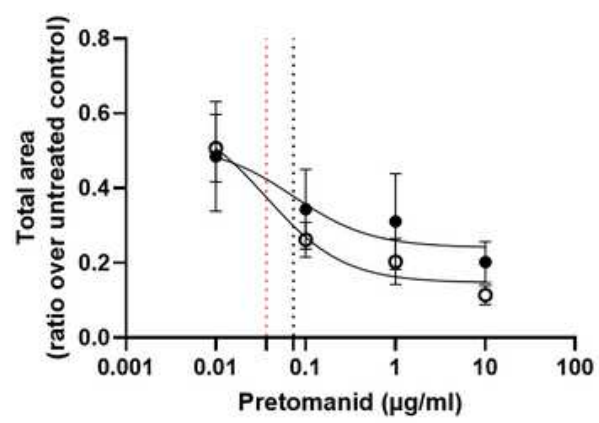

- planktonic

- cording

- cording

- planktonic

- cording

\section{Figure 5}

Dose response to antibiotics. Total area of aggregates in wells treated with antibiotics normalized to median of untreated controls ( $\mathrm{N}=33)$ were used to calculate IC50 values. Black dotted line represents IC50 value for planktonic and red dotted line IC50 value for cording phenotype as determined by nonlinear regression (inhibitor vs response) with 4 parameters $(\mathrm{A}, \mathrm{B})$ or 3 parameters $(\mathrm{C}-\mathrm{H})$. Data are presented as 
mean of three experiments $\pm S D$. Dotted lines 198 cross $x$-axes at the point representing IC50 value for planktonic (black line) and cording (red line) models.

A

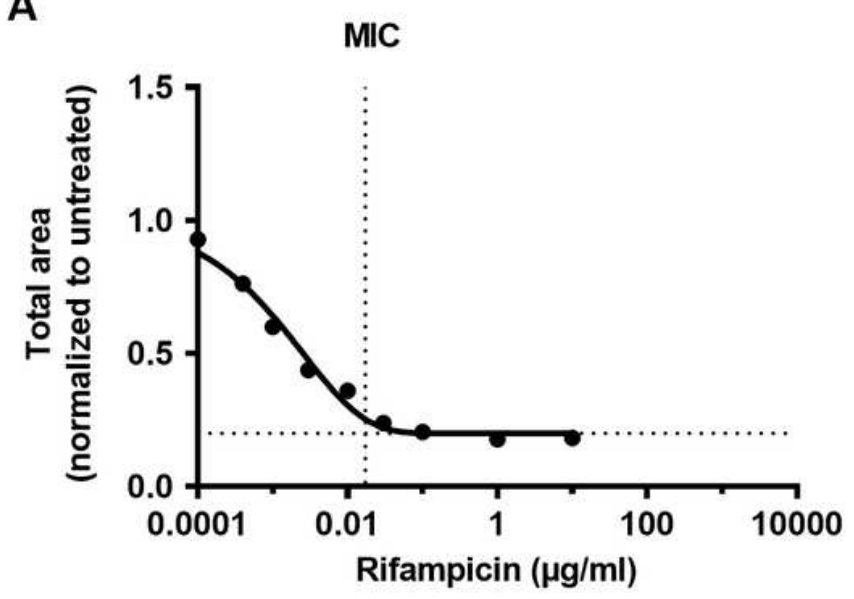

C

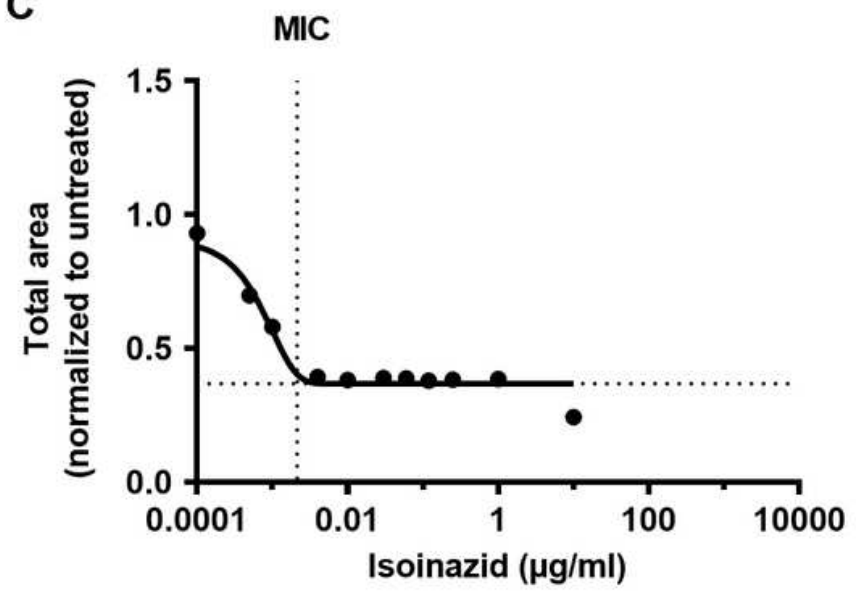

B

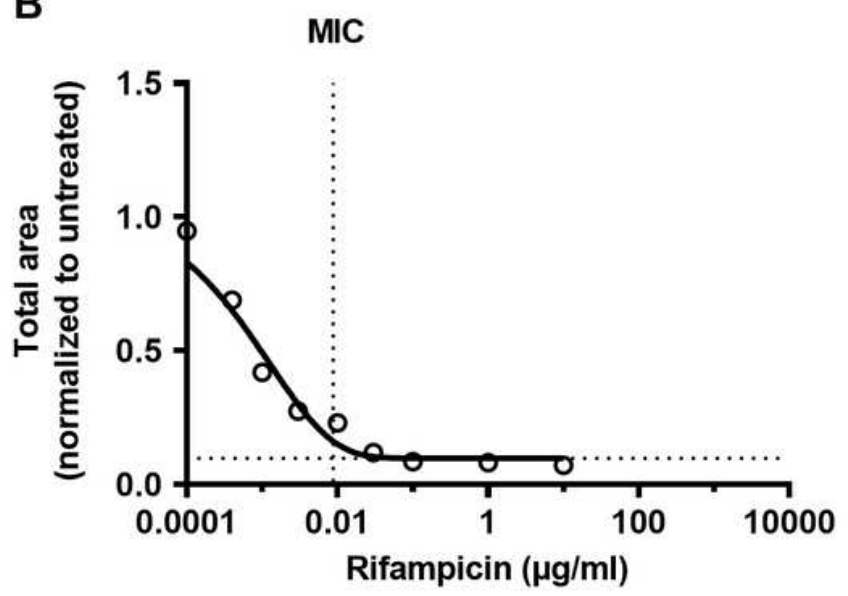

D

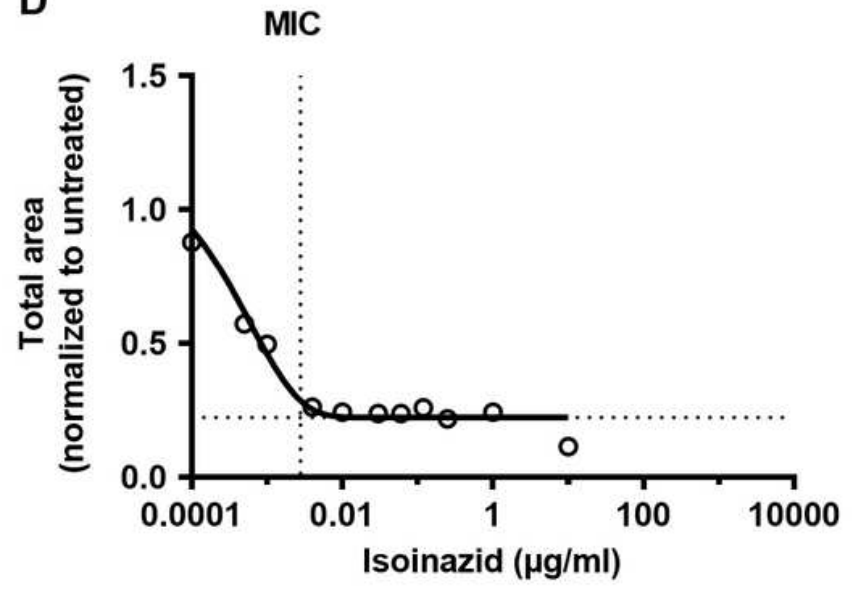

Figure 6

MIC values for rifampicin (RIF) and isoniazid (INH). Gompertz functions was used to calculate MIC values at day 5 based on total area of aggregates normalized to median of untreated controls $(\mathrm{N}=33)$ of the A), C) planktonic and B), D) cording model. 

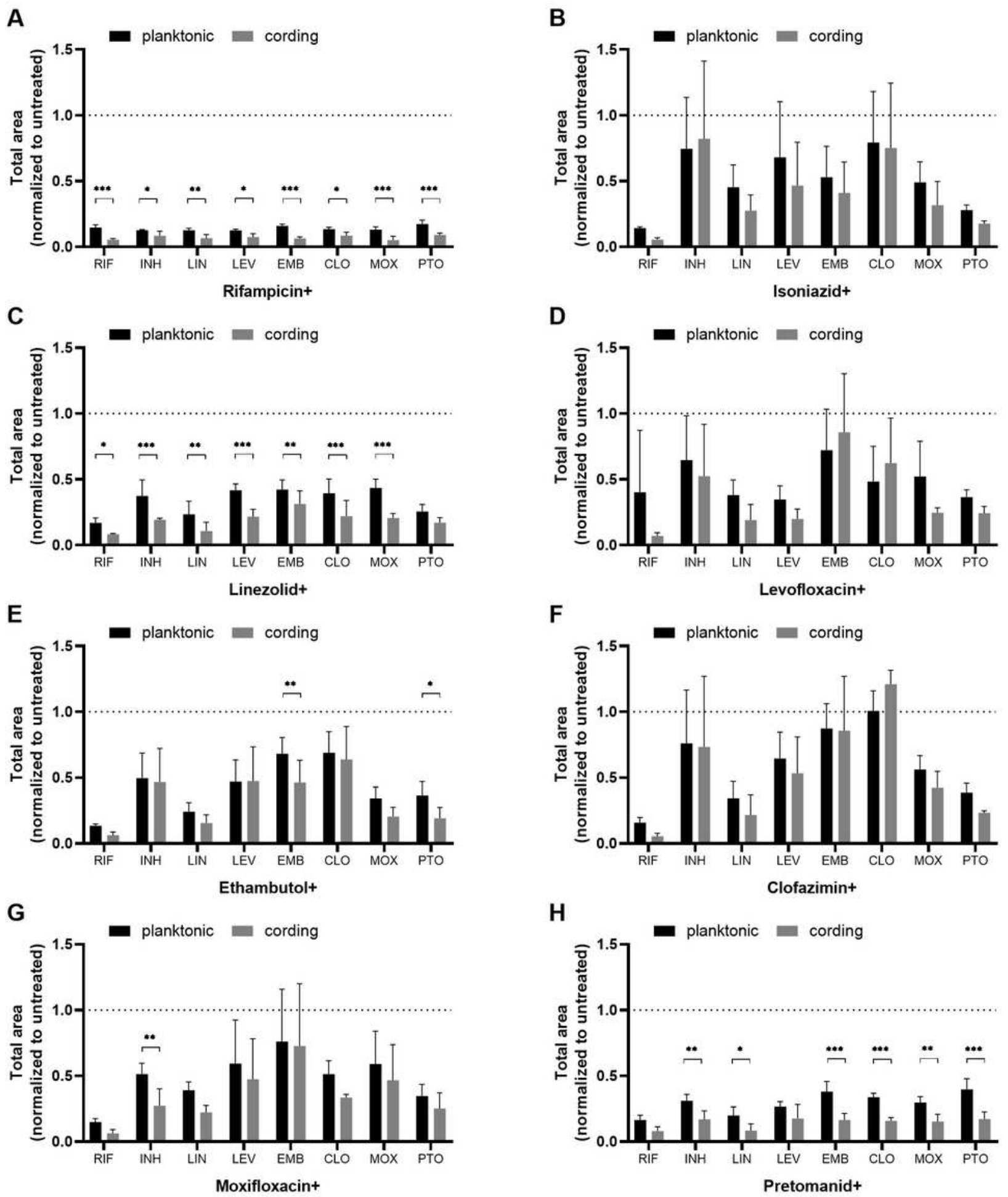

\section{Figure 7}

Inhibition of Rv growth by combination of antibiotics. H37Rv were treated by combination of two antibiotics at sub-inhibitory concentrations (details in Table 2). Total area of aggregates in wells treated with antibiotics normalized to median of untreated controls $(\mathrm{N}=33)$ were used in analysis. Data are presented as mean $\pm \mathrm{SD}(\mathrm{N}=3)$. 


\section{Supplementary Files}

This is a list of supplementary files associated with this preprint. Click to download.

- Additionalfile1TableS1.xlsx

- Additionalfile2Figs1.tif

- Additionalfile3Figs2.tif

- Additionalfile4FigS3.tif

- Additionalfile5Figs4.tif

- Additionalfile6FigS5.tif

- Additionalfile7TableS2.xIsx

- Additionalfile8MovieS1.mp4 